MPI-PhT/98-78

\title{
Correction to four-loop RG functions in the two-dimensional lattice $\mathrm{O}(n) \sigma$-model
}

\author{
Dong-Shin Shin \\ Max-Planck-Institut für Physik \\ - Werner-Heisenberg-Institut - \\ Föhringer Ring 6, 80805 Munich, Germany
}

\begin{abstract}
We report the result of our evaluation of the Feynman diagrams appearing in the determination of the four-loop renormalization group functions in the two-dimensional lattice $\mathrm{O}(n) \sigma$-model by Caracciolo and Pelissetto. In the list of the integrals used for the determination of those functions, we find that three entries were not correctly evaluated. We give the values for them corrected by us including those for all other integrals which we computed with higher precision. These results are then applied to revise the determination of the second analytic correction to correlation length $\xi$ and spin susceptibility $\chi$ by Caracciolo et al. as well as our determination of the mass gap by means of a finite volume technique where we explicitly made use of the four-loop $\beta$-function. In both cases we find sizeable changes in predictions. In the meantime there appeared a paper by Alles et al. where they revised one finite integral in the list of our corrected integrals. After having taken the new revised value into consideration, we found that there are no noticeable changes in the perturbative predictions of the present paper including the final conclusions.
\end{abstract}




\section{Introduction}

The two-dimensional non-linear $\mathrm{O}(n) \sigma$-model is interesting in theoretical physics in many respects. In condensed matter physics, the model can be applied to the study of ferromagnetic systems. In elementary particle physics, on the other hand, it serves as a good toy model for the investigation of the strong interactions since it shares with QCD many common properties.

The most interesting property of the $\sigma$-model from the view of particle physicists is that of asymptotic freedom which is predicted by perturbation theory. Besides this, the model shows many additional interesting properties. One believes a nonperturbative generation of a mass gap via dimensional transmutation which controls the exponential decay of correlation functions at large distances. From the existence of an infinite set of conserved non-local quantum charges [1, 2, 3, 4, one can show that there is no particle production in the theory and also derive "factorization equations" directly. These properties lead finally to the exact determination of the $S$-matrix up to a CDD-ambiguity [5, 6], assuming that the spectrum of the theory is known. By making use of the exact $S$-matrix and the thermodynamic Bethe ansatz, Hasenfratz and Niedermayer determined the ratio of the mass gap to the $\Lambda$-parameter $m / \Lambda$ of the theory exactly [7, 8]. If one formulates the $\sigma$-model on the lattice, it has a further advantage: due to low dimensionality it is simple to simulate numerically and can therefore be used in many cases for testing new ideas in lattice theories.

The property of asymptotic freedom is based on the validity of perturbation theory. One usually formulates perturbation theory on a non-symmetric trivial vacuum. The Mermin-Wagner theorem [9], however, tells us that in $\mathrm{O}(n) \sigma$-model the continuous $\mathrm{O}(n)$ symmetry can not be spontaneously broken in two dimensions. Therefore, the validity of the perturbation theory in infinite volume is in fact not guaranteed. This observation, together with the lack of clear asymptotic scaling in simulations, gives some authors [10, 11, 12] a motive for an argument that the nonlinear $\mathrm{O}(n) \sigma$-model has no mass gap and for any $n \geq 2$ it undergoes a KosterlitzThouless phase transition [13, 14] at a finite coupling $f_{c} \neq 0$.

Monte Carlo simulations allow in principle to explore the behavior of a model in all areas of the bare coupling. It is of general interest to connect Monte Carlo results with those of the perturbative renormalization group $(R G)$ which should dictate the scaling laws of the theory near the fixed point $f_{0}=0$. For a precise comparison with the Monte Carlo data, the computation of the RG functions at high orders in perturbation theory is desirable. Until now they are known up to four loops where the long and difficult four-loop computation was recently done by Caracciolo and Pelissetto [15]. These determinations of the four-loop $\beta$ - and $\gamma$-functions then allow us to compute the correlation length $\xi$ and spin susceptibility $\chi$ up to second analytic correction.

We applied an efficient coordinate space method for the evaluation of lattice Feynman diagrams described in detail in our paper [16 to compute the integrals 
listed in the paper by Caracciolo et al. more precisely. In most integrals, our results showed good agreement with those by Caracciolo et al. We, however, found that three integrals were not correctly evaluated. Since these errors turn out to cause sizable changes in the perturbative prediction to $\xi$ and $\chi$ as well as in our determination of the mass gap by a finite volume technique [17], we would like to report our results for the evaluation of the lattice integrals together with the corrections to the physical prediction to $\xi$ and $\chi$ as well as to our determination of the mass gap. Furthermore, the four-loop RG functions evaluated by Caracciolo et al. were applied in many papers 18, 19, 20, 21, 12 and it is necessary to revise them in accordance with our corrected values for the $R G$ functions.

We would like to stress that we checked only a minor part of the four-loop RG functions by Caracciolo et al., where we just reevaluated the integrals listed in that paper. We still do not know whether the other analytic formulas are correct. For the complete confirmation of the four-loop RG functions, it would take a while until we finish our own computation of them which is in progress [22].

The paper is organized as follows. In the next section we define the $\sigma$-model and give in this model the hitherto known results for the perturbative RG functions including the four-loop determination by Caracciolo et al. and its corrected version by us. Section 3 is then devoted to the reinvestigation of the physical quantities by making use of our corrected values for the RG functions. Finally, the explicit evaluations of lattice Feynman diagrams are presented in the Appendix.

\section{Computation of the RG functions}

We consider the non-linear $\mathrm{O}(n) \sigma$-model in two dimensions defined by the action

$$
S=\frac{1}{2 f_{0}} \int \mathrm{d}^{2} x\left[\partial_{\mu} q(x) \cdot \partial_{\mu} q(x)-2 h q_{n}(x)\right]
$$

where $q_{i}(x)$ with $i=1, \cdots, n$ is an $n$-component real scalar field of unit length $q(x)^{2}=1$ and $f_{0}$ denotes the bare coupling constant. $h$ is here a magnetic field which regularizes the infrared divergences and explicitly breaks the $\mathrm{O}(n)$ symmetry, lining up the field along the component $q_{n}$. At small coupling, one can then consider small fluctuations around the direction of the magnetic field. In this way, the perturbative expansions in $f_{0}$ can be obtained by setting

$$
q(x)=\left\{\pi_{1}(x), \cdots, \pi_{n-1}(x),\left[1-\sum_{\alpha=1}^{n-1} \pi_{\alpha}(x)^{2}\right]^{1 / 2}\right\}
$$

which solves the constraint $q(x)^{2}=1$. The regulator $h$ can be removed at the final stage of the computation. This is guarenteed by the conjecture of Elitzur [23], proved later by David order by order in perturbation theory [24], which states that in $\mathrm{O}(n) \sigma$-model, $\mathrm{O}(n)$ invariant correlation functions have a finite infrared limit in two dimensions. 
With this prescription, one can now start to compute the physically interesting quantities according to perturbation theory. Higher loop computations are however accompanied by ultraviolet divergences, which requires a renormalization. This can be achieved by introducing a regularization and redefining bare fields $\pi$ and coupling $f_{0}$ as renormalized ones, $\pi_{R}$ and $f$. In the dimensional regularization with dimension $d$, one sets $\pi_{R}=Z^{-1 / 2} \pi$ and $f=Z_{1}^{-1} f_{0} \mu^{d-2}$, where the $Z$ and $Z_{1}$, taken as a series in $f$, are adjusted so that the renormalized theory satisfies a chosen set of two independent conditions. Choosing $\mu$ as the scale of the renormalized theory, if one changes $\mu$, one has to change the renormalized fields and coupling accordingly, which is represented by the $R G$ equation

$$
\left\{\mu \frac{\partial}{\partial \mu}+\beta(f) \frac{\partial}{\partial f}+\frac{1}{2} n \gamma(f)+\left[\frac{1}{2} \gamma(f)+\frac{1}{f} \beta(f)\right] h \frac{\partial}{\partial h}\right\} G_{R}^{(n)}(p, f, \mu ; h)=0,
$$

where $G_{R}^{(n)}$ is the renormalized $n$-point correlation function for the $\pi_{R}$ field and $\beta(f)$ and $\gamma(f)$ are $\beta$ - and $\gamma$-functions respectively:

$$
\begin{aligned}
& \beta(f)=\mu \frac{\mathrm{d}}{\mathrm{d} \mu} f=-b_{1} f^{2}-b_{2} f^{3}-b_{3} f^{4}-b_{4} f^{5}+\mathcal{O}\left(f^{6}\right), \\
& \gamma(f)=\mu \frac{\mathrm{d}}{\mathrm{d} \mu} \ln Z=c_{1} f+c_{2} f^{2}+c_{3} f^{3}+c_{4} f^{4}+\mathcal{O}\left(f^{5}\right) .
\end{aligned}
$$

The coefficients $b_{1}, b_{2}$ and $c_{1}$ are universal in the sense that they do not depend on the renormalization scheme chosen and explicitly given by

$$
\begin{aligned}
& b_{1}=\frac{n-2}{2 \pi}, \\
& b_{2}=\frac{n-2}{(2 \pi)^{2}}, \\
& c_{1}=\frac{n-1}{2 \pi} .
\end{aligned}
$$

All other higher terms are, on the other hand, scheme dependent. In the minimal subtraction (MS) scheme, they were computed up to four loops [25, 26, 27, 28]:

$$
\begin{aligned}
& b_{3}=\frac{1}{4} \frac{n^{2}-4}{(2 \pi)^{3}} \\
& b_{4}=\frac{n-2}{(2 \pi)^{4}}\left[-\frac{1}{12}\left(n^{2}-22 n+34\right)+\frac{3}{2} \zeta(3)(n-3)\right] \\
& c_{2}=0 \\
& c_{3}=\frac{3}{32 \pi^{3}}(n-1)(n-2), \\
& c_{4}=\frac{1}{192 \pi^{4}}(n-1)(n-2)[4(5-n)+3(3-n) \zeta(3)] .
\end{aligned}
$$

with $\zeta(3) \simeq 1.2020569$ 
If one formulates the $\sigma$-model on the lattice, there are many different ways to define actions to regularize the theory. Here, we would like to consider the standard nearest-neighbor action

$$
S=-\frac{1}{f_{0}} \sum_{x, \mu} q(x) \cdot q(x+\hat{\mu})-h \sum_{x} q_{n}(x) .
$$

The RG equation on the lattice which is formally the same as Eq. (2.3), but written in terms of the bare $\beta$ - and $\gamma$-functions, is given by

$$
\left\{\hat{\beta}\left(f_{0}\right) \frac{\partial}{\partial f_{0}}-a \frac{\partial}{\partial a}-\frac{1}{2} n \hat{\gamma}\left(f_{0}\right)+\left[\frac{1}{2} \hat{\gamma}\left(f_{0}\right)+\frac{1}{f_{0}} \hat{\beta}\left(f_{0}\right)\right] h \frac{\partial}{\partial h}\right\} G_{L}^{(n)}\left(p, f_{0}, 1 / a ; h\right)=0,
$$

where $G_{L}^{(n)}$ is the lattice $n$-point correlation function for the $\pi$ field and the lattice $\beta$ - and $\gamma$-functions are given by

$$
\begin{aligned}
& \hat{\beta}\left(f_{0}\right)=-a \frac{\mathrm{d}}{\mathrm{d} a} f_{0}=-\hat{b}_{1} f_{0}^{2}-\hat{b}_{2} f_{0}^{3}-\hat{b}_{3} f_{0}^{4}-\hat{b}_{4} f_{0}^{5}+\mathcal{O}\left(f_{0}^{6}\right), \\
& \hat{\gamma}\left(f_{0}\right)=a \frac{\mathrm{d}}{\mathrm{d} a} \ln Z=\hat{c}_{1} f_{0}+\hat{c}_{2} f_{0}^{2}+\hat{c}_{3} f_{0}^{3}+\hat{c}_{4} f_{0}^{4}+\mathcal{O}\left(f_{0}^{5}\right) .
\end{aligned}
$$

The coefficients in this regularization are also known to four loops. Up to three loops, they are given by [29, 30, 31]

$$
\begin{aligned}
& \hat{b}_{1}=b_{1}, \\
& \hat{b}_{2}=b_{2}, \\
& \hat{b}_{3}=\frac{n-2}{(2 \pi)^{3}}\left[\left(\frac{1}{2}+\frac{1}{8} \pi^{2}-4 \pi^{2} G_{1}\right)(n-2)+1+\frac{1}{2} \pi-\frac{5}{24} \pi^{2}\right], \\
& \hat{c}_{1}=c_{1} \\
& \hat{c}_{2}=\frac{n-1}{8 \pi}, \\
& \hat{c}_{3}=\frac{n-1}{(2 \pi)^{3}}\left[\left(\frac{1}{2}-\frac{1}{8} \pi^{2}+4 \pi^{2} G_{1}\right)(n-2)+\frac{11}{24} \pi^{2}\right]
\end{aligned}
$$

with $G_{1} \simeq 0.0461636$. Recently, Caracciolo and Pelissetto [15] computed the quite demanding four-loop coefficients $\hat{b}_{4}$ and $\hat{c}_{4}$ :

$$
\begin{aligned}
\hat{b}_{4}= & \frac{n-2}{2 \pi}\left\{\frac{2 n-7}{96}+\frac{n^{2}-4 n+5}{32 \pi}+\frac{n}{16 \pi^{2}}+\frac{5 n-9}{16 \pi^{3}}+\frac{73 n-164}{96 \pi^{3}} \zeta(3)\right. \\
& -\frac{n-2}{2 \pi}(n-2+3 \pi) G_{1}-\frac{n-2}{\pi}(3-\pi) R+\frac{n-6}{24} J \\
& -\frac{n-2}{3} L_{1}+\frac{1}{8}(n-3)(n-2)\left(K-2 V_{3}\right) \\
& \left.+\frac{n-2}{2}\left[2 V_{1}+(n-2) V_{2}+4 V_{4}-2 V_{5}-2 V_{6}-16 W_{2}\right]\right\},
\end{aligned}
$$




$$
\begin{aligned}
\hat{c}_{4}= & \frac{n-1}{2 \pi}\left\{-\frac{5 n-21}{192}+\frac{(n-2)^{2}}{32 \pi}+\frac{3(n-2)}{32 \pi^{2}}+\frac{n-2}{16 \pi^{3}}\right. \\
& -\frac{n-2}{192 \pi^{3}}(6 n+37) \zeta(3)-\frac{n-2}{4 \pi}[2(n-2)-5 \pi] G_{1}+\frac{n-2}{2 \pi}(3-\pi) R \\
& -\frac{n-6}{48} J-\frac{n-2}{3} L_{1}-\frac{1}{16}(n-3)(n-2)\left(K-2 V_{3}\right) \\
& \left.+\frac{n-2}{4}\left[(n-3) V_{1}+2(n-2) V_{2}-4 V_{4}+2 V_{5}+2 V_{6}+16 W_{2}\right]\right\},
\end{aligned}
$$

where the integrals $G_{1}, R, J, K, L_{1}, V_{1}, \cdots, V_{6}, W_{1}$ and $W_{2}$ are defined in Appendix A and their numerical values evaluated by Caracciolo et al. are listed in the middle column of Table 1 .

\begin{tabular}{c||l|l}
\hline Integrals & Values I & Values II \\
\hline$G_{1}$ & 0.0461636 & $0.04616362923(1)$ \\
$R$ & 0.0148430 & $0.014842965985741(1)$ \\
$J$ & 0.1366198 & $0.136619772367581(1)$ \\
$K$ & 0.095887 & $0.09588764425(1)$ \\
$L_{1}$ & 0.0029334 & $0.002933289310(1)$ \\
$V_{1}$ & 0.016961 & $0.016961078576(1)$ \\
$V_{2}$ & -0.00114 & $-0.0011381683(1)$ \\
$V_{3}$ & 0.07243 & $0.07243631946(1)$ \\
$V_{4}$ & -0.0013125 & $-0.002624929(1)$ \\
$V_{5}$ & 0.010063 & $0.0100630371(1)$ \\
$V_{6}$ & 0.017507 & $0.017506946(1)$ \\
$W_{1}$ & -0.0296860 & $-0.007421482994(1)$ \\
$W_{2}$ & 0.00221 & $0.0006923019(1)$ \\
\hline
\end{tabular}

Table 1: Numerical evaluations of the lattice integrals appearing in the four-loop $\beta$ - and $\gamma$-functions. The numbers in the middle column (Values I) are the evaluations by Caracciolo et al., while the numbers in the right column (Values II) are those by the author.

By applying the coordinate space method for the evaluation of lattice Feynman diagrams described in detail in our paper [16], we reevaluated all integrals in Table 1 with higher precision. We show our results in the right column of the same table. As we see in Table 1, we reproduce most values by Caracciolo et al. with a minor precision mistake for the constant $L_{1}$. However, for three integrals $V_{4}, W_{1}$ and $W_{2}$, our numbers are different from those by Caracciolo et al., where the difference in $V_{4}$ can be accounted for by a factor of 2 .

\footnotetext{
${ }^{1}$ Recently, Alles et al. [35] revised the value for $W_{2}$. We improved its numerical precision to get $W_{2}=0.0021225468(1)$ [36].
} 
We remark that Caracciolo et al. checked their determination of the four-loop RG functions by the technique of $1 / n$ expansion. In the limit of large $n$, Eqs. (2.24) and (2.25) reduce to

$$
\begin{aligned}
& \frac{\hat{b}_{4}}{n^{3}}=\frac{1}{2 \pi^{2}}\left[\frac{1}{32}-\frac{G_{1}}{2}+\frac{\pi}{8}\left(K+4 V_{2}-2 V_{3}\right)\right]+\mathcal{O}\left(\frac{1}{n}\right) \\
& \frac{\hat{c}_{4}}{n^{3}}=\frac{1}{2 \pi}\left[\frac{1}{32 \pi}-\frac{1}{32 \pi^{3}} \zeta(3)-\frac{G_{1}}{2 \pi}-\frac{K}{16}+\frac{V_{1}}{4}+\frac{V_{2}}{2}+\frac{V_{3}}{8}\right]+\mathcal{O}\left(\frac{1}{n}\right) .
\end{aligned}
$$

Eqs. (2.26) and (2.27) agree with the predictions by the $1 / n$ expansion in the given order [15]. However, as we see in the above equations, this method can not control all integrals in Table 1, merely those of $G_{1}, K, V_{1}, V_{2}$ and $V_{3}$.

There was, on the other hand, a further check on the RG functions when we determined a mass gap 17 by applying a perturbative computation of the mass gap $M(L)$ in finite volume $L$. In converting our computation of $M(L)$ on the lattice to the renormalized MS scheme, we made there an explicit use of the four-loop $\beta$ function. From the constraint that we should formally have a free theory in the $\mathrm{O}(2)$ model with dimensional regularization, we get a relationship for the perturbative $M(L)$ to satisfy. In this case, we could however confirm only the integral $J$.

\section{Application to physical quantities}

In this section we present the application of the perturbative determination of the RG functions in the previous section to the physical predictions, explicitly the analytic correction to the correlation length $\xi$ and spin susceptibility $\chi$ as well as the determination of a mass gap through a perturbative computation of a finite volume mass gap $M(L)$. We first consider the application to the analytic correction to $\xi$ and $\chi$.

\subsection{Perturbative evaluation of $\xi$ and $\chi$}

The determination of the four-loop $\beta$ - and $\gamma$-functions makes it possible to compute $\xi$ and $\chi$ up to second analytic correction. We discuss here the numerical evaluation of the analytic corrections to $\xi$ and $\chi$ and also to the other relevant quantities. We first consider the correlation length $\xi$.

The correlation length is a physical quantity and correspondingly satisfies the RG equation:

$$
\left[\hat{\beta}\left(f_{0}\right) \frac{\partial}{\partial f_{0}}-a \frac{\partial}{\partial a}\right] \xi\left(f_{0}, a\right)=0
$$

We may then define the RG invariant parameter as

$$
\xi / a=C_{\xi}\left(\hat{b}_{1} f_{0}\right)^{\hat{b}_{2} / \hat{b}_{1}^{2}} \exp \left(\frac{1}{\hat{b}_{1} f_{0}}\right) \bar{\xi}\left(f_{0}\right),
$$




$$
\bar{\xi}\left(f_{0}\right)=\exp \left[\int_{0}^{f_{0}} \mathrm{~d} x\left(\frac{1}{\hat{\beta}(x)}+\frac{1}{\hat{b}_{1} x^{2}}-\frac{\hat{b}_{2}}{\hat{b}_{1}^{2} x}\right)\right]
$$

which satisfies Eq. (3.1) order by order in perturbation theory. The constant $C_{\xi}$ is non-perturbative and its value depends on the explicit definition of the correlation length. For the isovector exponential correlation length $\xi_{V}^{(\exp )}$, which controls the large distance behaviour of the two-point function $\langle q(x) \cdot q(0)\rangle$, an exact determination was achieved by Hasenfratz and Niedermayer [7, 8] using the thermodynamic Bethe ansatz:

$$
C_{\xi_{V}^{(\exp )}}=\left(\frac{\mathrm{e}}{8}\right)^{1 /(n-2)} \Gamma\left(1+\frac{1}{n-2}\right) 2^{-5 / 2} \exp \left[-\frac{\pi}{2(n-2)}\right]
$$

An alternative definition with respect to the isovector correlation function $\langle q(x) \cdot q(0)\rangle$ is the second-moment correlation length

$$
\xi_{V}^{(2)}=\sqrt{\frac{\sum_{x}|x|^{2}\langle q(x) \cdot q(0)\rangle}{\sum_{x}\langle q(x) \cdot q(0)\rangle}} .
$$

There are also other definitions with respect to higher isospin channels, e.g. the exponential correlation length related to the isotensor correlation function $\left\langle[q(x) \cdot q(0)]^{2}-1 / n\right\rangle$ and the corresponding second-moment correlation length.

The function $\bar{\xi}\left(f_{0}\right)$ of Eq. (3.3) is regular at $f_{0}=0$ and may be expanded in $f_{0}$ :

$$
\bar{\xi}\left(f_{0}\right)=1+A_{1} f_{0}+A_{2} f_{0}^{2}+\mathcal{O}\left(f_{0}^{3}\right),
$$

where

$$
\begin{aligned}
& A_{1}=\frac{\hat{b}_{1} \hat{b}_{3}-\hat{b}_{2}^{2}}{\hat{b}_{1}^{3}} \\
& A_{2}=\frac{\left(\hat{b}_{2}^{2}-\hat{b}_{1} \hat{b}_{3}\right)^{2}+\hat{b}_{1}^{2}\left(\hat{b}_{2}^{3}-2 \hat{b}_{1} \hat{b}_{2} \hat{b}_{3}+\hat{b}_{1}^{2} \hat{b}_{4}\right)}{2 \hat{b}_{1}^{6}} .
\end{aligned}
$$

Inserting $\hat{b}_{1}, \hat{b}_{2}, \hat{b}_{3}$ and $\hat{b}_{4}$ from Eqs. (2.18)-(2.20) and (2.24) in Eqs. (3.7) and (3.8), we obtain for $A_{1}$ and $A_{2}$

$$
\begin{aligned}
A_{1}= & \frac{1}{n-2}\left[\left(\frac{1}{4 \pi}+\frac{\pi}{16}-2 \pi G_{1}\right)(n-2)+\frac{1}{4}-\frac{5 \pi}{48}\right], \\
A_{2}= & \frac{1}{192(n-2)^{2}}\left[\frac{\pi^{2}}{24}(3 n-11)^{2}+\pi\left(4 n^{2}-19 n+17\right)\right. \\
& \left.+6 n^{3}-39 n^{2}+95 n-76\right] \\
& +\frac{1}{32 \pi^{2}(n-2)}[7 n-16+2 \pi(n-1)]+\frac{1}{96 \pi^{2}} \frac{73 n-164}{n-2} \zeta(3)
\end{aligned}
$$




$$
\begin{aligned}
& +\frac{G_{1}}{24(n-2)}\left[-12(n-2)(n-3)-12 \pi(3 n-5)-(3 n-11) \pi^{2}\right] \\
& +2 \pi^{2} G_{1}^{2}-(3-\pi) R+\frac{\pi}{24} \frac{n-6}{n-2} J-\frac{\pi}{3} L_{1}+\frac{\pi}{8}(n-3)\left(K-2 V_{3}\right) \\
& +\frac{1}{2} \pi\left[2 V_{1}+(n-2) V_{2}+4 V_{4}-2 V_{5}-2 V_{6}-16 W_{2}\right] .
\end{aligned}
$$

Now, if we substitute for the constants in Eqs. (3.9) and (3.10) the values in Table 1 evaluated by us, we get for $A_{2}$ an estimation which is different from that by Caracciolo et al.:

$$
\begin{aligned}
& A_{1} \simeq \frac{1}{n-2}(-0.0490-0.0141 n) \\
& A_{2} \simeq \frac{1}{(n-2)^{2}}\left(0.188-0.122 n+0.0405 n^{2}-0.0129 n^{3}\right)
\end{aligned}
$$

It is to be remarked that if $n$ increases, the difference with the determination by Caracciolo et al. becomes small, which is of course to be expected since the four-loop coefficient is correct in the large $n$ limit.

Next, we would like to compute the analytic correction to the isovector spin susceptibility $\chi_{V}=\sum_{x}\langle q(x) \cdot q(0)\rangle$. It satisfies the following RG equation:

$$
\left[\hat{\beta}\left(f_{0}\right) \frac{\partial}{\partial f_{0}}-a \frac{\partial}{\partial a}+\hat{\gamma}\left(f_{0}\right)\right] \chi_{V}\left(f_{0}, a\right)=0
$$

We may then define the RG invariant parameter as

$$
\begin{aligned}
\chi_{V} / a^{2} & =C_{\chi}\left(\hat{b}_{1} f_{0}\right)^{\left(2 \hat{b}_{2}+\hat{c}_{1} \hat{b}_{1}\right) / \hat{b}_{1}^{2}} \exp \left(\frac{2}{\hat{b}_{1} f_{0}}\right) \bar{\chi}\left(f_{0}\right), \\
\bar{\chi}\left(f_{0}\right) & =\exp \left[\int_{0}^{f_{0}} \mathrm{~d} x\left(\frac{2}{\hat{\beta}(x)}+\frac{2}{\hat{b}_{1} x^{2}}-\frac{2 \hat{b}_{2}}{\hat{b}_{1}^{2} x}-\frac{\hat{\gamma}(x)}{\hat{\beta}(x)}-\frac{\hat{c}_{1}}{\hat{b}_{1} x}\right)\right] .
\end{aligned}
$$

Eq. (3.14) with Eq. (3.15) satisfies the RG equation (3.13) order by order in perturbation theory. Contrary to $C_{\xi}$ in Eq. (3.2), there is no exact expression for the non-perturbative constant $C_{\chi}$ at present. It is however known in the large $n$ limit [32, 33]:

$$
C_{\chi}=\frac{\pi}{16}\left[1+\frac{1}{n}\left(4+3 \gamma_{C}-\pi-3 \gamma_{E}-7 \ln 2\right)+\mathcal{O}\left(\frac{1}{n^{2}}\right)\right]
$$

where $\gamma_{E}$ is Euler's constant and $\gamma_{C} \simeq 0.4861007$.

The function $\bar{\chi}\left(f_{0}\right)$ of Eq. (3.15) is well-defined at $f_{0}=0$ and may be expanded in $f_{0}$ :

$$
\bar{\chi}\left(f_{0}\right)=1+B_{1} f_{0}+B_{2} f_{0}^{2}+\mathcal{O}\left(f_{0}^{3}\right)
$$


with

$$
\begin{aligned}
B_{1}= & \frac{2 \hat{b}_{1} \hat{b}_{3}-2 \hat{b}_{2}^{2}+\hat{b}_{1}^{2} \hat{c}_{2}-\hat{b}_{1} \hat{b}_{2} \hat{c}_{1}}{\hat{b}_{1}^{3}} \\
B_{2}= & \frac{\left[2 \hat{b}_{2}^{2}+\hat{b}_{1} \hat{b}_{2} \hat{c}_{1}-\hat{b}_{1}\left(2 \hat{b}_{3}+\hat{b}_{1} \hat{c}_{2}\right)\right]^{2}}{2 \hat{b}_{1}^{6}} \\
& +\frac{2 \hat{b}_{2}^{3}+\hat{b}_{1} \hat{b}_{2}^{2} \hat{c}_{1}-\hat{b}_{1} \hat{b}_{2}\left(4 \hat{b}_{3}+\hat{b}_{1} \hat{c}_{2}\right)+\hat{b}_{1}^{2}\left(2 \hat{b}_{4}-\hat{b}_{3} \hat{c}_{1}+\hat{b}_{1} \hat{c}_{3}\right)}{2 \hat{b}_{1}^{4}} .
\end{aligned}
$$

Inserting $\hat{b}_{1}, \cdots, \hat{b}_{4}$ and $\hat{c}_{1}, \cdots, \hat{c}_{3}$ from Eqs. (2.18)-(2.24) in Eqs. (3.18) and (3.19), we obtain for $B_{1}$ and $B_{2}$

$$
\begin{aligned}
B_{1}= & \frac{1}{2 \pi(n-2)}\left[\frac{1}{2} \pi(n+1)-1+\frac{1}{4} \pi^{2}\left(n-\frac{11}{3}\right)-8 \pi^{2}(n-2) G_{1}\right], \\
B_{2}= & \frac{1}{(n-2)^{2}}\left[\frac{\pi^{2}}{1152}(3 n-11)^{2}+\frac{\pi}{96}\left(7 n^{2}-30 n+17\right)\right. \\
& +\frac{1}{96}(n-1)\left(3 n^{2}-13 n+51\right) \\
& \left.-\frac{1}{8 \pi}(2 n-1)+\frac{1}{8 \pi^{2}}\left(3 n^{2}-13 n+15\right)\right]+\frac{1}{48 \pi^{2}} \frac{73 n-164}{n-2} \zeta(3) \\
& +\frac{G_{1}}{6(n-2)}\left[6(3 n-4)-6 \pi(4 n-5)-(3 n-11) \pi^{2}\right]+8 \pi^{2} G_{1}^{2} \\
& -2(3-\pi) R+\frac{\pi}{12} \frac{n-6}{n-2} J-\frac{2 \pi}{3} L_{1}+\frac{\pi}{4}(n-3)\left(K-2 V_{3}\right) \\
& +\pi\left[2 V_{1}+(n-2) V_{2}+4 V_{4}-2 V_{5}-2 V_{6}-16 W_{2}\right] .
\end{aligned}
$$

Now, if we substitute for the constants in Eqs. (3.20) and (3.21) our values in Table 1, we obtain

$$
\begin{aligned}
& B_{1} \simeq \frac{1}{n-2}(-0.1888+0.0626 n) \\
& B_{2} \simeq \frac{1}{(n-2)^{2}}\left(0.4191-0.2688 n+0.0517 n^{2}-0.0108 n^{3}\right) .
\end{aligned}
$$

Again, for $B_{2}$ we get a prediction which is different from that by Caracciolo et al.

Let us also consider the ratio $\chi_{V} / \xi^{2}$ which has an advantage of being possible to compute an additional analytic correction. Writing

$$
\frac{\chi_{V}}{\xi^{2}}=R_{V}\left(\frac{2 \pi}{n-2} \frac{1}{f_{0}}\right)^{-(n-1) /(n-2)}\left(1+\sum_{i=1}^{\infty} C_{i} f_{0}^{i}\right),
$$

where $R_{V}=C_{\chi} / C_{\xi}^{2}$ is a non-perturbative universal constant, the expansion coefficients $C_{1}, C_{2}$ and $C_{3}$ have the expressions

$$
C_{1}=\frac{1}{4 \pi} \frac{n-1}{n-2}(\pi-2),
$$




$$
\begin{aligned}
C_{2}= & \frac{n-1}{(n-2)^{2}}\left[(n-2)^{2} G_{1}-\frac{1}{96}\left(3 n^{2}-23 n+31\right)+\frac{n-1}{8 \pi^{2}}-\frac{2 n-3}{8 \pi}\right], \\
C_{3}= & \frac{n-1}{(n-2)^{3}}\left[-\frac{1}{384}\left(10 n^{3}-79 n^{2}+186 n-137\right)\right. \\
& +\frac{1}{192 \pi}(2 n-3)\left(3 n^{2}-26 n+37\right) \\
& \left.+\frac{1}{32 \pi^{2}}(n-1)(3 n-5)-\frac{1}{48 \pi^{3}}\left(3 n^{3}-18 n^{2}+38 n-27\right)\right] \\
& \frac{n-1}{n-2}\left\{-\frac{1}{192 \pi^{3}}\left(2 n^{2}+57 n-134\right) \zeta(3)\right. \\
& \left.-\frac{1}{48}(n-6) J+\frac{G_{1}}{4 \pi}[(5 n-9) \pi-2(2 n-3)]\right\} \\
& +(n-1)\left[\frac{1}{2 \pi}(3-\pi) R-\frac{n-3}{16}\left(K-2 V_{3}\right)\right. \\
& \left.+\frac{n-7}{12} V_{1}+\frac{1}{2}\left(-2 V_{4}+V_{5}+V_{6}+8 W_{2}\right)\right] .
\end{aligned}
$$

The numerical evaluations for these coefficients with our values in Table 1 yield

$$
\begin{aligned}
& C_{1} \simeq \frac{n-1}{n-2} 0.0908 \\
& C_{2} \simeq \frac{n-1}{(n-2)^{2}}\left(-0.0316-0.0120 n+0.0149 n^{2}\right) \\
& C_{3} \simeq \frac{n-1}{(n-2)^{3}}\left(0.0578-0.0564 n+0.0273 n^{2}-0.0149 n^{3}+0.0041 n^{4}\right) .
\end{aligned}
$$

As expected, for the expansion coefficient $C_{3}$ our determination does not agree with the estimation by Caracciolo et al.

Finally, we would like to consider the spin- $k$ susceptibility [15] defined as

$$
\chi^{(k)}=\sum_{x}\left\langle Y_{k}^{\alpha_{1}, \cdots, \alpha_{k}}(0) Y_{k}^{\alpha_{1}, \cdots, \alpha_{k}}(x)\right\rangle
$$

where

$$
Y_{k}^{\alpha_{1}, \cdots, \alpha_{k}}=q_{\alpha_{1}} \cdots q_{\alpha_{k}}-\text { "Traces" }
$$

and the "Traces" must be such that $Y_{k}^{\alpha_{1}, \cdots, \alpha_{k}}$ is symmetric and traceless. Then, from the standard RG arguments one has

$$
\chi^{(k)}=C_{\chi}^{(k)} \exp \left(\frac{4 \pi}{n-2} \frac{1}{f_{0}}\right)\left(\frac{2 \pi}{n-2} \frac{1}{f_{0}}\right)^{-[2+k(n+k-2)] /(n-2)}\left[1+\sum_{i=1}^{\infty} B_{i}^{(k)} f_{0}^{i}\right]
$$

with a non-universal constant $C_{\chi}^{(k)}$. By including the ratio

$$
\frac{\chi^{(k)}}{\xi^{2}}=R^{(k)}\left(\frac{2 \pi}{n-2} \frac{1}{f_{0}}\right)^{-k(n+k-2) /(n-2)}\left[1+\sum_{i=1}^{\infty} C_{i}^{(k)} f_{0}^{i}\right]
$$




\begin{tabular}{cclccl}
\hline $\mathrm{n}$ & $\beta$ & \multicolumn{1}{c}{$\xi$} & \multicolumn{1}{c}{$\mathcal{R}^{(2)}$} & \multicolumn{1}{c}{$\mathcal{R}^{(3)}$} & \multicolumn{1}{c}{$\mathcal{R}^{(4)}$} \\
\hline 3 & 1.85 & $89.16(21)$ & $0.743(2)$ & $0.781(2)$ & $0.822(2)$ \\
3 & 2.25 & $1049(7)$ & $0.861(6)$ & $0.897(6)$ & $0.928(6)$ \\
3 & 2.60 & $8569(92)$ & $0.901(10)$ & $0.934(10)$ & $0.958(10)$ \\
3 & 3.00 & $94.6(1.6) \cdot 10^{3}$ & $0.930(16)$ & $0.959(16)$ & $0.977(17)$ \\
4 & 2.50 & $34.85(9)$ & $0.911(2)$ & $0.930(2)$ & $0.949(2)$ \\
4 & 2.80 & $86.07(37)$ & $0.927(4)$ & $0.945(4)$ & $0.960(4)$ \\
8 & 5.80 & $33.41(8)$ & $0.985(2)$ & $0.990(2)$ & $0.994(2)$ \\
\hline
\end{tabular}

Table 2: Ratio of Monte Carlo results to the theoretical perturbative predictions at two, three and four loops for the correlation length $\xi$ in the $\mathrm{O}(3), \mathrm{O}(4)$ and $\mathrm{O}(8)$ model evaluated for various values of $\beta$.

and its expansion coefficients

$$
\begin{aligned}
C_{1}^{(k)} & =\frac{1}{4 \pi} \frac{k(n+k-2)}{n-2}(\pi-2), \\
C_{2}^{(k)} & =\frac{1}{2}\left[c_{1}^{(k)}\right]^{2}+\frac{k(n+k-2)}{n-2}\left[(n-2) G_{1}-\frac{1}{8 \pi}-\frac{1}{96}(3 n-14)\right],
\end{aligned}
$$

one gets for the expansion coefficients

$$
\begin{aligned}
& B_{1}^{(k)}=2 A_{1}+C_{1}^{(k)} \\
& B_{2}^{(k)}=2 A_{2}+A_{1}^{2}+C_{2}^{(k)}+2 A_{1} C_{1}^{(k)}
\end{aligned}
$$

which have the numerical values

$$
\begin{aligned}
B_{1}^{(k)} \simeq & \frac{1}{n-2}[-0.0980-0.0283 n+0.0908 k(k+n-2)], \\
B_{2}^{(k)} \simeq & \frac{1}{(n-2)^{2}}\left[0.37866-0.2428 n+0.08115 n^{2}-0.0257 n^{3}\right. \\
& +k(k+n-2)\left(-0.0363-0.0187 n+0.0149 n^{2}\right) \\
& \left.+0.0041 k^{2}(k+n-2)^{2}\right] .
\end{aligned}
$$

Here, our value of $B_{2}^{(k)}$ is not in agreement with that by Caracciolo et al.

Now, it is interesting to compare the theory with "experiment". We would like to take the correlation length and compute the ratio

$$
\mathcal{R}^{(l)}(\beta)=\frac{\xi_{\mathrm{MC}}(\beta)}{\xi_{\mathrm{th}}^{(l)}(\beta)}
$$

where $\xi_{\mathrm{MC}}(\beta)$ is the Monte Carlo value for the correlation length given as a function of the inverse bare coupling $\beta=1 / f_{0}$ and $\xi_{\text {th }}^{(l)}(\beta)$ is the theoretical $l$-loop prediction 
from Eqs. (3.2)-(3.4). In Table 2 we show determinations of $\mathcal{R}^{(l)}$ for some selected values of $n$ and $\beta$ by inserting our new corrected coefficient of the four-loop $\beta$ function. Compared to the old estimations by Caracciolo et al., the correction of the four-loop $\beta$-function causes to reduce the four-loop contribution to the perturbative correlation length in all of the considered cases, where the difference is maximum in the $\mathrm{O}(3)$ model for $\beta=1.85$ and becomes small if $\beta$ or/and $n$ increase. It follows that, in every case, the four-loop contributions to the correlation length are smaller than Caracciolo et al. have estimated, and so the discrepancies between the theoretical four-loop prediction and "experiment" are larger than the estimation by Caracciolo et al. Explicitly, at the largest $\beta$ values investigated, they are $2.3 \%$ for the $\mathrm{O}(3)$ model, $4.0 \%$ for the $\mathrm{O}(4)$ model and $0.6 \%$ for the $\mathrm{O}(8)$ model and there still remains some space for higher loop contributions.

\subsection{Determination of a mass gap by finite volume method}

In our work [17 on the determination of a mass gap $m$ by applying the perturbative computation of the mass gap $M(L)$ in finite volume $L$, we made an explicit use of the four-loop $\beta$-function in converting our computation of $M(L)$ regularized on a lattice to the renormalized MS-scheme. Since the four-loop coefficient has to be changed, our prediction for the mass gap has to be corrected accordingly, and so we would like to present here the modifications caused by the change of the $\beta$-function.

To the determination of the physical infinite volume mass gap $m$ from the finite volume mass gap $M(L)$, we applied two different methods: one method was the determination by matching the behavior of the finite volume mass gap $M(L)$ evaluated at small $L$ with that of the mass shift $\delta_{0} \equiv[M(L)-m] / m$ known at large $L$ and the other was by means of a coupling $\bar{g}^{2}(L)$ running with $L$. We analyzed both methods again by inserting our new corrected value of the $\beta$-function.

In the case of the determination of the mass gap by applying the mass shift, we concluded that the method is not stable and so it is difficult to make a reasonable estimation for the mass gap by this method. After we substituted our new corrected value of the four-loop $\beta$-function, we see substantial changes, but the whole picture including the final conclusion does not improve the situation. Therefore, we do not want to present here the evaluation of the mass gap by this method. As for the determination of the mass gap by means of running coupling, we however see interesting effects through the new corrected value and therefore we would like to discuss in the following the new evaluation of the mass gap by this method in detail.

In Ref. [17], we introduced a coupling $\bar{g}^{2}(L)$ running with volume $L$ defined by

$$
\bar{g}^{2}(L)=2 M(L) L /(n-1)
$$

where $M(L)$ is a mass gap in finite volume which we computed to three-loop order

\footnotetext{
${ }^{2}$ The running coupling $\bar{g}^{2}(L)$ was first defined in Ref. [34].
} 
in perturbation theory. For the coefficients of $\beta$-function defined by

$$
\tilde{\beta}\left(\bar{g}^{2}\right) \equiv-L \frac{\partial \bar{g}^{2}}{\partial L}=-\bar{g}^{2} \sum_{l=1}^{\infty} \tilde{b}_{l}\left(\bar{g}^{2}\right)^{l},
$$

we found

$$
\begin{aligned}
& \tilde{b}_{1}=b_{1}, \\
& \tilde{b}_{2}=b_{2}, \\
& \tilde{b}_{3}=\frac{(n-1)(n-2)}{(2 \pi)^{3}}, \\
& \tilde{b}_{4}=\frac{1}{4} \frac{n-2}{(2 \pi)^{4}}\left[(n-2)^{3} \chi_{1}+(n-2)^{2} \chi_{2}+(n-2) \chi_{3}+\chi_{4}\right] .
\end{aligned}
$$

The explicit expressions for the constants $\chi_{1}, \cdots, \chi_{4}$ can be found in appendix B of Ref. [17], where the values for $t_{1}, t_{2}$ and $t_{3}$ in their expressions have now to be modified by our new values:

$$
\hat{b}_{4}=\frac{n-2}{(2 \pi)^{4}}\left[(n-2)^{2} t_{1}+(n-2) t_{2}+t_{3}\right]
$$

with $^{3}$

$$
\begin{aligned}
& t_{1}=-1.015069687473002(1) \\
& t_{2}=-5.9168085(1) \\
& t_{3}=-9.0937547(1) .
\end{aligned}
$$

By inserting these corrected numbers into the expressions for $\chi_{1}, \cdots, \chi_{4}$, we get the numerical values

$$
\begin{aligned}
& \chi_{1}=-1.20(1), \quad \chi_{2}=-3.63(1), \\
& \chi_{3}=23.6(1), \\
& \chi_{4}=-5.2123414(1) .
\end{aligned}
$$

The $\Lambda$-parameter in this scheme is given by

$$
\begin{aligned}
\Lambda_{F V} & =\frac{1}{L}\left(b_{1} \bar{g}^{2}\right)^{-b_{2} / b_{1}^{2}} e^{-1 /\left(b_{1} \bar{g}^{2}\right)} \cdot \tilde{\lambda}\left(\bar{g}^{2}\right), \\
\tilde{\lambda}\left(\bar{g}^{2}\right) & =\exp \left[-\int_{0}^{\bar{g}^{2}} d x\left(\frac{1}{\tilde{\beta}(x)}+\frac{1}{b_{1} x^{2}}-\frac{b_{2}}{b_{1}^{2} x}\right)\right]
\end{aligned}
$$

which has the following perturbative expansion up to order $\mathcal{O}\left(\bar{g}^{4}\right)$ :

$$
\Lambda_{F V}^{(2)}=\frac{1}{L}\left(b_{1} \bar{g}^{2}\right)^{-b_{2} / b_{1}^{2}} e^{-1 /\left(b_{1} \bar{g}^{2}\right)},
$$

\footnotetext{
${ }^{3}$ In the evaluation of $t_{1}, t_{2}$ and $t_{3}$ we took the revised value for $W_{2}$ in Ref. 35 into consideration.
} 


$$
\begin{aligned}
\Lambda_{F V}^{(3)}= & \frac{1}{L}\left(b_{1} \bar{g}^{2}\right)^{-b_{2} / b_{1}^{2}} e^{-1 /\left(b_{1} \bar{g}^{2}\right)}\left\{1+\frac{b_{2}^{2}-b_{1} \tilde{b}_{3}}{b_{1}^{3}} \bar{g}^{2}\right\} \\
\Lambda_{F V}^{(4)}= & \frac{1}{L}\left(b_{1} \bar{g}^{2}\right)^{-b_{2} / b_{1}^{2}} e^{-1 /\left(b_{1} \bar{g}^{2}\right)}\left\{1+\frac{b_{2}^{2}-b_{1} \tilde{b}_{3}}{b_{1}^{3}} \bar{g}^{2}\right. \\
& \left.+\frac{b_{2}^{4}-b_{1}^{2} b_{2}^{3}+2 b_{1}^{3} b_{2} \tilde{b}_{3}-2 b_{1} b_{2}^{2} \tilde{b}_{3}+b_{1}^{2} \tilde{b}_{3}^{2}-b_{1}^{4} \tilde{b}_{4}}{2 b_{1}^{6}} \bar{g}^{4}\right\} .
\end{aligned}
$$

If we insert the running couplings $\bar{g}^{2}(L)$ from Table 1 of Ref. [17], measured for given $L$ in units of the mass gap $m$ in $\mathrm{O}(3)$ model, in Eqs. (3.57)-(3.59), we obtain new values for the ratio $\Lambda_{F V} / m$ in 2-, 3- and 4-loop approximations which are listed in Table 3 and also illustrated in Fig. 11.

The new effect which mainly arises by substituting our corrected value for the four-loop $\beta$-function is that, compared to the old diagram with the value by Caracciolo et al., for large couplings the new four-loop estimations for $\Lambda_{F V} / m$ become smaller, so that the four-loop curve in Fig. 1 shifts underneath. As a result, the order of 3- and 4-loop curves changes. However, when the coupling goes to zero, the difference with the old diagram becomes smaller and smaller, and at the smallest coupling considered, it is practically zero. From this observation we can conclude that the changes in $\beta$-function influence only at large couplings and for small couplings, which are indeed the region of our main interest, we have the same picture as the earlier investigation with the value by Caracciolo et al. Therefore, as in the case with the old four-loop $\beta$-function, our determination of the mass gap converges to the value predicted by Hasenfratz and Niedermayer very well.

To give a further impression on the systematic errors, we also consider an alternative definition of $n$-loop approximations to $\Lambda_{F V}$; instead of expanding $\tilde{\lambda}\left(\bar{g}^{2}\right)$ of Eq. (3.56) in the coupling $\bar{g}^{2}$, we insert the perturbative coefficients of $\tilde{\beta}(x)$ in $\tilde{\lambda}\left(\bar{g}^{2}\right)$ and integrate this exactly. If we apply the coefficients of the $\beta$-function calculated up to 4-loop order, we obtain the following approximations to $\Lambda_{F V}$ at 2-, 3- and 4-loop levels which are a little modified from Eqs.(3.57)-(3.59):

$$
\begin{aligned}
\bar{\Lambda}_{F V}^{(2)}= & \frac{1}{L}\left(b_{1} \bar{g}^{2}\right)^{-b_{2} / b_{1}^{2}} e^{-1 /\left(b_{1} \bar{g}^{2}\right)} \\
& \cdot \exp \left[-\int_{0}^{\bar{g}^{2}} d x\left(\frac{1}{x^{2}\left(b_{1}+b_{2} x\right)}+\frac{1}{b_{1} x^{2}}-\frac{b_{2}}{b_{1}^{2} x}\right)\right] \\
\bar{\Lambda}_{F V}^{(3)}= & \frac{1}{L}\left(b_{1} \bar{g}^{2}\right)^{-b_{2} / b_{1}^{2}} e^{-1 /\left(b_{1} \bar{g}^{2}\right)} \\
& \cdot \exp \left[-\int_{0}^{\bar{g}^{2}} d x\left(\frac{1}{x^{2}\left(b_{1}+b_{2} x+\tilde{b}_{3} x^{2}\right)}+\frac{1}{b_{1} x^{2}}-\frac{b_{2}}{b_{1}^{2} x}\right)\right] \\
\bar{\Lambda}_{F V}^{(4)}= & \frac{1}{L}\left(b_{1} \bar{g}^{2}\right)^{-b_{2} / b_{1}^{2}} e^{-1 /\left(b_{1} \bar{g}^{2}\right)} \\
& \cdot \exp \left[-\int_{0}^{\bar{g}^{2}} d x\left(\frac{1}{x^{2}\left(b_{1}+b_{2} x+\tilde{b}_{3} x^{2}+\tilde{b}_{4} x^{3}\right)}+\frac{1}{b_{1} x^{2}}-\frac{b_{2}}{b_{1}^{2} x}\right)\right] .
\end{aligned}
$$




\begin{tabular}{cccc}
\hline $\bar{g}^{2}(L)$ & $\Lambda_{F V}^{(2)} / m$ & $\Lambda_{F V}^{(3)} / m$ & $\Lambda_{F V}^{(4)} / m$ \\
\hline 0.5372 & $0.0511(8)$ & $0.0467(6)$ & $0.0463(17)$ \\
0.5747 & $0.0512(7)$ & $0.0465(6)$ & $0.0460(17)$ \\
0.6060 & $0.0514(7)$ & $0.0464(6)$ & $0.0459(15)$ \\
0.6553 & $0.0518(6)$ & $0.0464(5)$ & $0.0457(15)$ \\
0.6970 & $0.0520(6)$ & $0.0462(5)$ & $0.0455(15)$ \\
0.7383 & $0.0525(6)$ & $0.0463(5)$ & $0.0455(13)$ \\
0.7646 & $0.0526(6)$ & $0.0462(5)$ & $0.0453(13)$ \\
0.8166 & $0.0532(6)$ & $0.0463(5)$ & $0.0453(11)$ \\
0.9176 & $0.0552(5)$ & $0.0471(5)$ & $0.0458(10)$ \\
1.0595 & $0.0574(4)$ & $0.0478(3)$ & $0.0459(8)$ \\
1.2680 & $0.0628(1)$ & $0.0502(2)$ & $0.0473(8)$ \\
\hline
\end{tabular}

Table 3: Two-, three- and four-loop approximations to $\Lambda_{F V} / m$ in the $\mathrm{O}(3)$ model

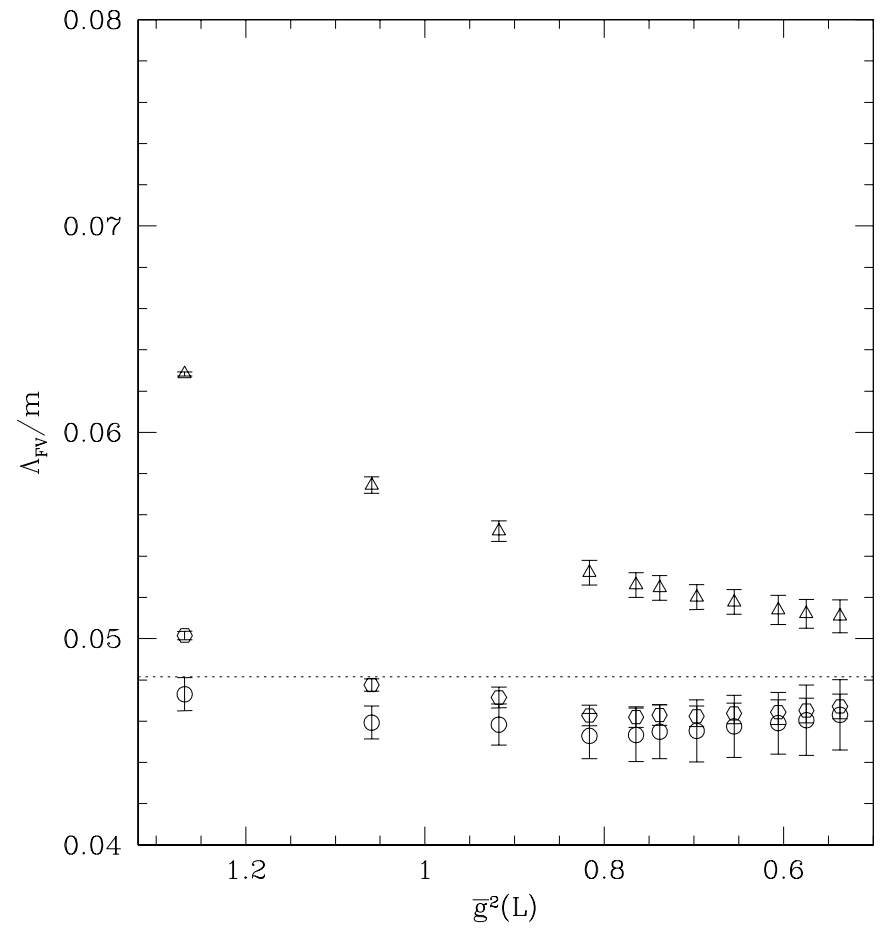

Figure 1: Two-, three- and four-loop approximations to $\Lambda_{F V} / m$ in the $\mathrm{O}(3)$ model from Table 3. The triangular points show the 2-loop approximation to the $\Lambda$ parameter, the hexagonal points the 3-loop and the circular points the 4-loop approximation. The dotted line is the value of Hasenfratz et al. $\left(\Lambda_{F V} / m=0.04815 \cdots\right)$. 
Insertion of the data in Table 1 of Ref. [17] in these equations gives the numbers in Table 1 which are also plotted in Fig. 2. In this case, we have exactly the same phenomenon as the former investigation. Compared to the old figure with the fourloop $\beta$-function value by Caracciolo et al., the four-loop curve for $\Lambda_{F V} / m$ at large couplings shifts underneath in this case as well, so that the splitting of 3- and 4loop curves becomes larger. However, for small couplings which are actually our interesting region, the difference between two figures, one figure with the new $\beta$ function value by us and the other with the old value by Caracciolo et al., vanishes. Therefore, also in this investigation with the modified expansion to the $\Lambda$-parameter, we can conclude in the same way as the earlier investigation with the old value of the $\beta$-function: all three curves converge to the value predicted by Hasenfratz et al.

We also want to discuss the changes of the diagrams through the correction of the $\beta$-function in $\mathrm{O}(4)$ model. By substituting the new value of the $\beta$-function in Eqs. (3.57)-(3.59), we obtain the corrected results for $\Lambda_{F V} / m$ which are written in Table 5 and also illustrated in Fig. 3. As far as the modification of the four-loop curve is concerned, Fig. 3 shows exactly the same behavior as Fig. 11: as a result of the shift of the four-loop curve underneath, the order of 3- and 4-loop curves changes, but for small couplings the difference between the old diagram and the new one vanishes and we have the same argument with respect to the final conclusion.

As for the determination of $\Lambda_{F V} / m$ by means of the modified definition of the perturbative $\Lambda_{F V}$ in $\mathrm{O}(4)$ model, we obtain the results which are written in Table 6 and also plotted in Fig. 1. In this case, we see exactly the same phenomenon as Fig. 2 which leads to the conclusion that for small couplings all three curves converge to the value by Hasenfratz et al. very well.

Summarizing the whole discussions up to now, we conclude that the correction of the four-loop $\beta$-function has an influence on our earlier investigation for the determination of the mass gap only at large couplings, while in the actually interesting region of small couplings it provides practically no changes. Therefore, our conclusions with the old value of the $\beta$-function by Caracciolo et al. remain valid and we can say that in all of the investigated cases our determinations of the mass gap with

the corrected value of the four-loop $\beta$-function by us agree with those by Hasenfratz et al. very well.

\section{Acknowledgement}

I greatly acknowledge Peter Weisz for many useful discussions as well as for reading this manuscript. 


\begin{tabular}{cccc}
\hline $\bar{g}^{2}(L)$ & $\bar{\Lambda}_{F V}^{(2)} / m$ & $\bar{\Lambda}_{F V}^{(3)} / m$ & $\bar{\Lambda}_{F V}^{(4)} / m$ \\
\hline 0.5372 & $0.0555(8)$ & $0.0474(6)$ & $0.0465(17)$ \\
0.5747 & $0.0559(7)$ & $0.0473(6)$ & $0.0462(17)$ \\
0.6060 & $0.0563(7)$ & $0.0473(6)$ & $0.0461(15)$ \\
0.6553 & $0.0572(6)$ & $0.0474(5)$ & $0.0460(15)$ \\
0.6970 & $0.0578(6)$ & $0.0474(5)$ & $0.0459(15)$ \\
0.7383 & $0.0586(6)$ & $0.0476(5)$ & $0.0459(23)$ \\
0.7646 & $0.0590(6)$ & $0.0476(5)$ & $0.0458(13)$ \\
0.8166 & $0.0601(6)$ & $0.0479(5)$ & $0.0458(11)$ \\
0.9176 & $0.0633(5)$ & $0.0492(5)$ & $0.0466(10)$ \\
1.0595 & $0.0671(4)$ & $0.0505(3)$ & $0.0472(8)$ \\
1.2680 & $0.0755(1)$ & $0.0544(2)$ & $0.0496(8)$ \\
\hline
\end{tabular}

Table 4: Alternative 2-, 3- and 4-loop approximations to $\Lambda_{F V} / m$ in the $\mathrm{O}(3)$ model

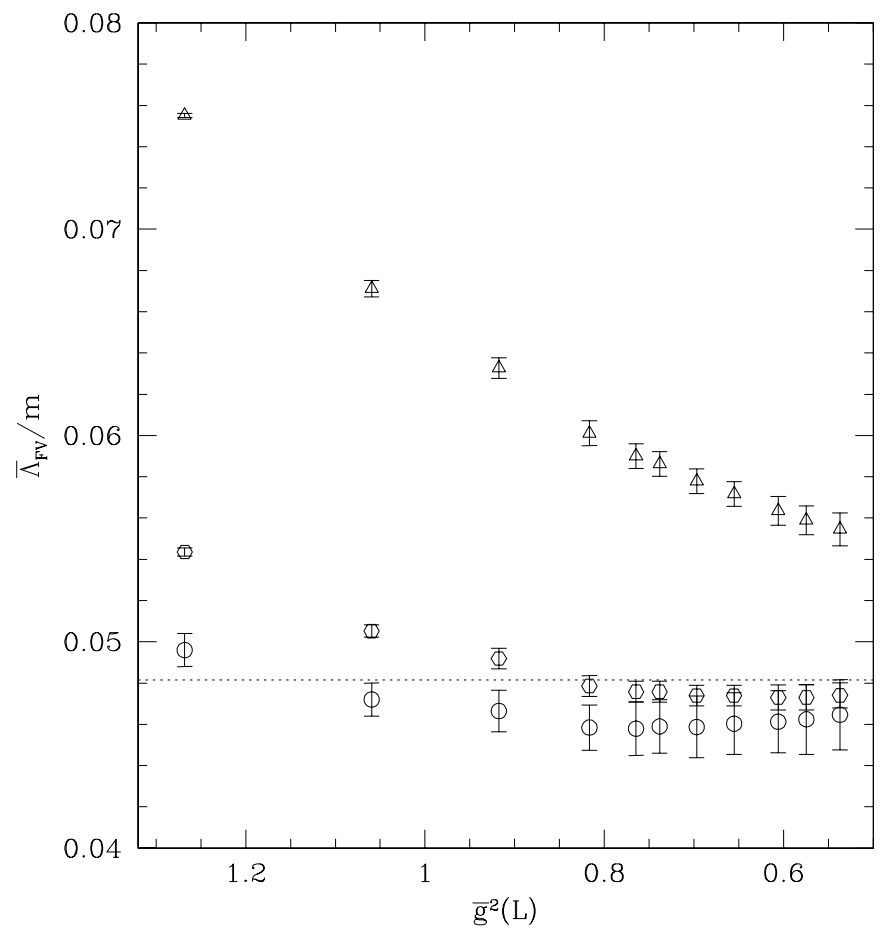

Figure 2: Two-, three- and four-loop approximations to $\Lambda_{F V} / m$ in the $\mathrm{O}(3)$ model from Table 4 . The arrangement of the points and the dotted line is the same as in Fig. 1. 


\begin{tabular}{cccc}
\hline $\bar{g}^{2}(L)$ & $\Lambda_{F V}^{(2)} / m$ & $\Lambda_{F V}^{(3)} / m$ & $\Lambda_{F V}^{(4)} / m$ \\
\hline 0.863 & $0.0795(7)$ & $0.0722(7)$ & $0.0717(16)$ \\
1.011 & $0.0817(7)$ & $0.0729(6)$ & $0.0722(15)$ \\
1.228 & $0.0844(5)$ & $0.0734(6)$ & $0.0723(12)$ \\
1.584 & $0.0880(5)$ & $0.0733(6)$ & $0.0713(10)$ \\
2.309 & $0.0928(4)$ & $0.0701(4)$ & $0.0657(9)$ \\
4.132 & $0.0853(4)$ & $0.0479(4)$ & $0.0351(9)$ \\
\hline
\end{tabular}

Table 5: Two-, three- and four-loop approximations to $\Lambda_{F V} / m$ in the $\mathrm{O}(4)$ model

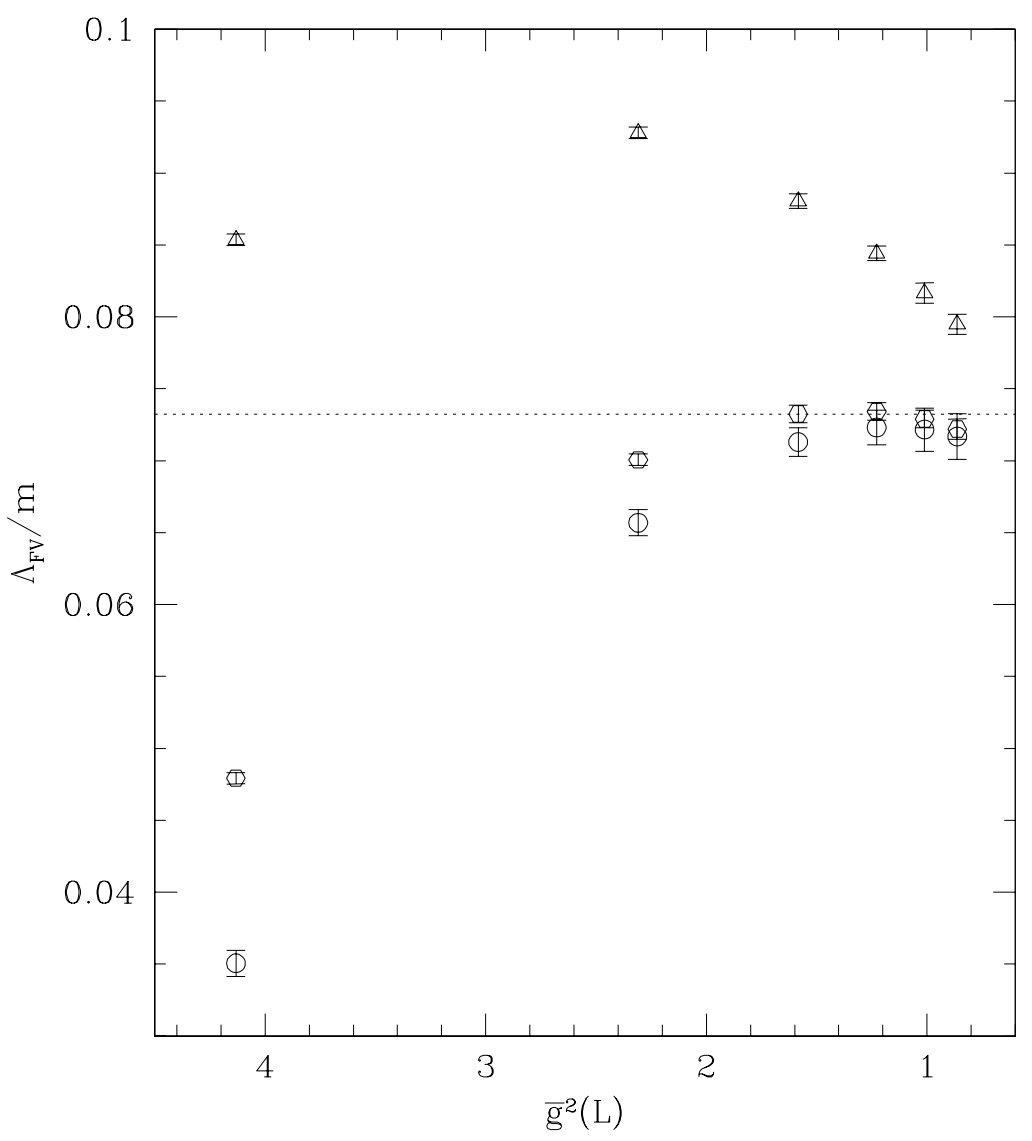

Figure 3: Two-, three- and four-loop approximations to $\Lambda_{F V} / m$ in the $\mathrm{O}(4)$ model from Table 5. The arrangement of the points is the same as in Fig. 11. The dotted line is the value of Hasenfratz et al. $\left(\Lambda_{F V} / m=0.07321 \cdots\right)$. 


\begin{tabular}{cccc}
\hline $\bar{g}^{2}(L)$ & $\bar{\Lambda}_{F V}^{(2)} / m$ & $\bar{\Lambda}_{F V}^{(3)} / m$ & $\bar{\Lambda}_{F V}^{(4)} / m$ \\
\hline 0.863 & $0.0830(7)$ & $0.0733(7)$ & $0.0720(16)$ \\
1.011 & $0.0859(7)$ & $0.0744(6)$ & $0.0726(15)$ \\
1.228 & $0.0898(5)$ & $0.0757(6)$ & $0.0731(12)$ \\
1.584 & $0.0952(5)$ & $0.0771(6)$ & $0.0730(10)$ \\
2.309 & $0.1035(4)$ & $0.0780(4)$ & $0.0709(9)$ \\
4.132 & $0.1024(4)$ & $0.0678(4)$ & $0.0561(9)$ \\
\hline
\end{tabular}

Table 6: Alternative 2-, 3- and 4-loop approximations to $\Lambda_{F V} / m$ in the $\mathrm{O}(4)$ model

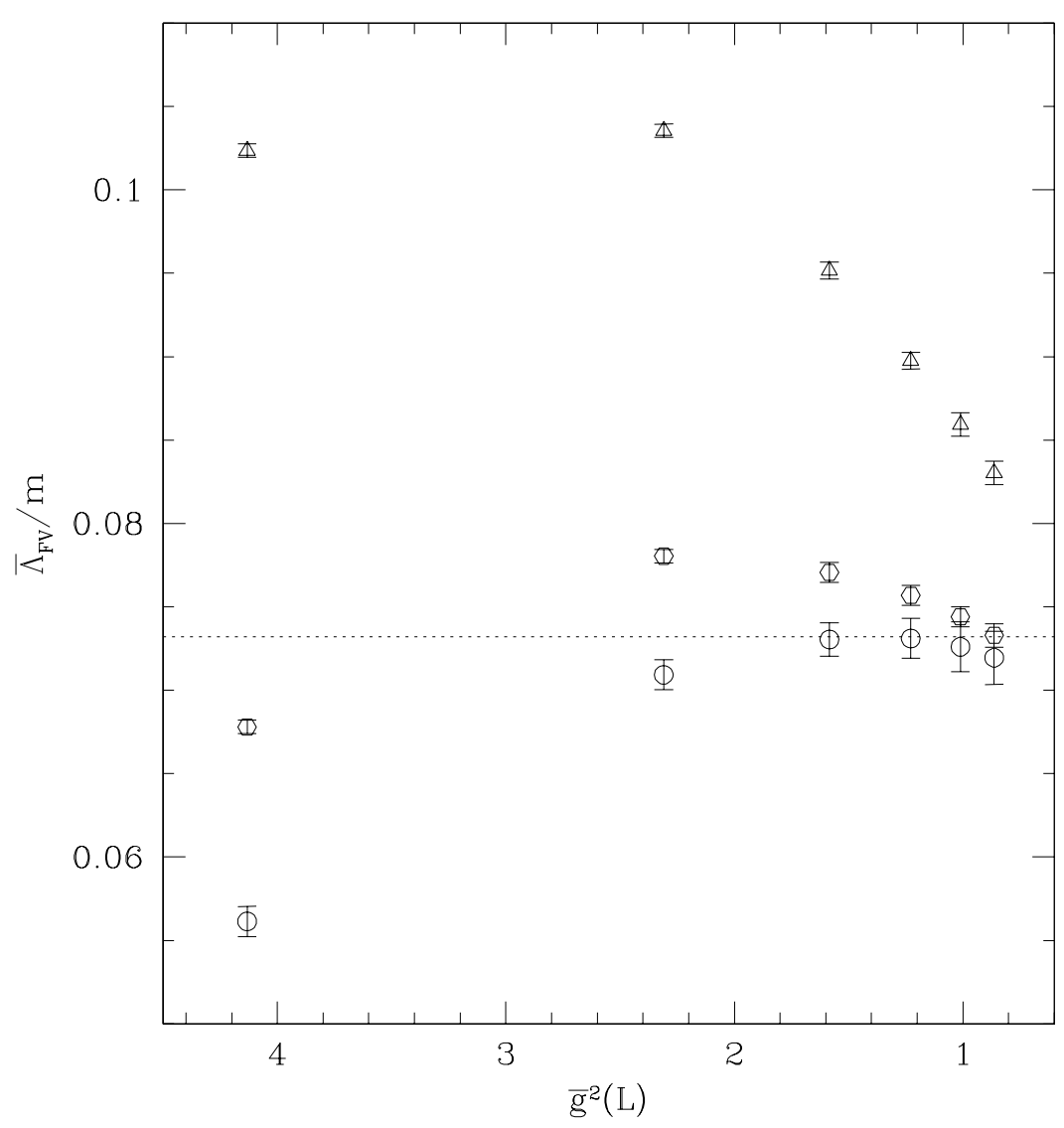

Figure 4: Two-, three- and four-loop approximations to $\Lambda_{F V} / m$ in the $\mathrm{O}(4)$ model from Table 6. The arrangement of the points and the dotted line is the same as in Fig. 通. 


\section{A Evaluation of lattice Feynman diagrams}

In this appendix we define the lattice integrals appearing in Table 1 of section 2 and show how they can be evaluated.

\section{A.1 Evaluation of $W_{2}$}

$W_{2}$ is defined as a finite part of an infrared divergent integral:

$$
W_{2}=\lim _{h \rightarrow 0}\left[\bar{W}_{2}(h)-\frac{1}{6} K(h)^{3}+\left(\frac{1}{8}-\frac{1}{8 \pi}\right) K(h)^{2}-\left(\frac{1}{32}+\frac{1}{16 \pi^{2}}-\frac{1}{16 \pi}-\frac{R}{2}\right) K(h)\right]
$$

with

$$
\bar{W}_{2}(h)=\int_{q r s t} \frac{\sum_{\mu, \nu} \sin q_{\mu} \sin r_{\nu} \sin s_{\mu} \sin t_{\nu}}{\left(\hat{q}^{2}+h\right)\left(\hat{r}^{2}+h\right)\left(\hat{s}^{2}+h\right)\left(\hat{t}^{2}+h\right)} \frac{1}{(\widehat{q+r})^{2}+h} \bar{\delta}(q+r+s+t),
$$

where $\bar{\delta}(q)$ stands for a modified form of $\delta$-function $(2 \pi)^{2} \delta(q)$ and $\int_{q}$ for an abbreviated symbol of the integral sign $\int_{-\pi}^{\pi} \frac{\mathrm{d}^{2} q}{(2 \pi)^{2}}$. The quantity $R$ is a finite integral which can be evaluated exactly

$$
R=\lim _{h \rightarrow 0}\left[h \int_{q r s} \frac{1}{\left(\hat{q}^{2}+h\right)\left(\hat{r}^{2}+h\right)\left(\hat{s}^{2}+h\right)} \bar{\delta}(q+r+s)\right]=\frac{1}{24 \pi^{2}} \psi^{\prime}\left(\frac{1}{3}\right)-\frac{1}{36}
$$

with $\psi(z)=\mathrm{d} \ln \Gamma(z) / \mathrm{d} z$, while the function $K(h)$ is a divergent integral for small $h$

$$
K(h)=\int_{p} \frac{1}{\hat{p}^{2}+h}=-\frac{1}{4 \pi} \ln \frac{h}{32}+\mathcal{O}(h \ln h)
$$

with $\hat{p}^{2} \equiv \sum_{\mu} \hat{p}_{\mu}^{2}, \hat{p}_{\mu} \equiv 2 \sin \left(p_{\mu} / 2\right)$ and $\mu=0,1$. Our aim is to evaluate the integral $\bar{W}_{2}(h)$ in the limit $h \rightarrow 0$ to confirm the logarithmic terms in Eq. (A.1) and to determine the constant $W_{2}$ at the end.

Observing that Eq. (A.2) can be represented by the following symmetric form

$$
\bar{W}_{2}(h)=\int_{p} \frac{1}{\hat{p}^{2}+h} \sum_{\mu, \nu} F_{\mu \nu}(p, h)^{2}
$$

with

$$
F_{\mu \nu}(p, h)=\int_{q r} \frac{\sin q_{\mu} \sin r_{\nu}}{\left(\hat{q}^{2}+h\right)\left(\hat{r}^{2}+h\right)} \bar{\delta}(p+q+r),
$$

we try to evaluate Eq. (A.5) by dividing into the following two integrals:

$$
\bar{W}_{2}(h)=I_{1}(h)+I_{2}(h),
$$


where

$$
\begin{aligned}
I_{1}(h) & =\int_{p} \frac{1}{\hat{p}^{2}+h} \sum_{\mu, \nu} F_{\mu \nu}(p, 0)^{2}, \\
I_{2}(h) & =\int_{p} \frac{1}{\hat{p}^{2}+h} \sum_{\mu, \nu}\left[F_{\mu \nu}(p, h)^{2}-F_{\mu \nu}(p, 0)^{2}\right] .
\end{aligned}
$$

Considering, at first, the integral $I_{1}(h)$, we transform the function $F_{\mu \nu}(p, 0)$ to position space to study how it behaves for small $p$ :

$$
F_{\mu \nu}(p, 0)=-\frac{1}{4} \sum_{x} \mathrm{e}^{i p x} x_{\mu} x_{\nu} H(x)^{2},
$$

where $H(x)$ is defined by

$$
H(x)=\int_{p} \mathrm{e}^{i p x} \ln \hat{p}^{2} .
$$

For large $x$, Eq. (A.11) shows the behavior $\lim _{x \rightarrow \infty} H(x) \sim \frac{1}{x^{2}}$, from which we can find out that $F_{\mu \nu}(p, 0)$ diverges logarithmically for $p \rightarrow 0$. We then break up Eq. (A.10) into converging and diverging part

$F_{\mu \nu}(p, 0)=-\frac{1}{4}\left[\bar{F}_{\mu \nu}(p, 0)+\frac{1}{4 \pi} \sum_{x} \mathrm{e}^{i p x}\left(\partial_{\mu}+\partial_{\mu}^{*}\right)\left(\partial_{\nu}+\partial_{\nu}^{*}\right) G(x)-\frac{1}{2 \pi} \delta_{\mu \nu} \sum_{x} \mathrm{e}^{i p x} H(x)\right]$,

where the free propagator $G(x)$ including its properties is discussed in detail in Ref. [16 and $\bar{F}_{\mu \nu}(p, 0)$ is a well-defined function in the limit $p \rightarrow 0$, constructed by subtracting from Eq. (A.10) the leading square terms in large $x$ :

$$
\bar{F}_{\mu \nu}(p, 0)=\sum_{x} \mathrm{e}^{i p x}\left[x_{\mu} x_{\nu} H(x)^{2}-\frac{1}{4 \pi}\left(\partial_{\mu}+\partial_{\mu}^{*}\right)\left(\partial_{\nu}+\partial_{\nu}^{*}\right) G(x)+\frac{1}{2 \pi} \delta_{\mu \nu} H(x)\right] .
$$

Next, we separate the $p=0$ part of Eq. (A.13) to introduce a function $\tilde{F}_{\mu \nu}(p, 0)$ which vanishes for $p=0$ :

$$
\bar{F}_{\mu \nu}(p, 0)=\tilde{F}_{\mu \nu}(p, 0)+\delta_{\mu \nu} C
$$

with

$$
\begin{aligned}
\tilde{F}_{\mu \nu}(p, 0) & =\sum_{x}\left(\mathrm{e}^{i p x}-1\right)\left[x_{\mu} x_{\nu} H(x)^{2}-\frac{1}{\pi} \partial_{\mu} \partial_{\nu}^{*} G(x)+\frac{\delta_{\mu \nu}}{2 \pi} H(x)\right], \\
C & =\frac{1}{2} \sum_{x}\left[x^{2} H(x)^{2}-\frac{1}{4 \pi} \sum_{\mu}\left(\partial_{\mu}+\partial_{\mu}^{*}\right)^{2} G(x)+\frac{1}{\pi} H(x)\right] .
\end{aligned}
$$

The numerical value of $C$, which can be computed very precisely by applying the position space method [16], is $0.369899 \cdots$. 
Substituting Eq. (A.12), together with Eq. (A.14), in Eq. (A.8) and going back to momentum space, we have

$$
I_{1}(h)=\frac{1}{16} \int_{p} \frac{1}{\hat{p}^{2}+h} \sum_{\mu, \nu}\left[\tilde{F}_{\mu \nu}(p, 0)-\frac{1}{\pi} \frac{\sin p_{\mu} \sin p_{\nu}}{\hat{p}^{2}}-\frac{1}{2 \pi} \delta_{\mu \nu} \ln \hat{p}^{2}+\delta_{\mu \nu} C\right]^{2}
$$

with

$$
\tilde{F}_{\mu \nu}(p, 0)=-4 \int_{q r} \frac{\sin q_{\mu} \sin r_{\nu}}{\hat{q}^{2} \hat{r}^{2}} \bar{\delta}(p+q+r)+\frac{1}{\pi} \frac{\sin p_{\mu} \sin p_{\nu}}{\hat{p}^{2}}+\frac{1}{2 \pi} \delta_{\mu \nu} \ln \hat{p}^{2}-\delta_{\mu \nu} C .
$$

Expanding the square, Eq. (A.17) produces the following ten integrals which we can evaluate separately:

$$
\begin{aligned}
I_{1}(h)= & \frac{1}{16}\left[2 C^{2} K(h)-\frac{2}{\pi} C I_{1}^{a}(h)+\frac{1}{2 \pi^{2}} I_{1}^{b}(h)-\frac{2}{\pi} C I_{1}^{c}(h)+\frac{1}{\pi^{2}} I_{1}^{d}(h)\right. \\
& \left.+\frac{1}{\pi^{2}} I_{1}^{e}(h)+2 C I_{1}^{f}(h)-\frac{1}{\pi} I_{1}^{g}(h)-\frac{2}{\pi} I_{1}^{h}(h)+I_{1}^{i}(h)\right]
\end{aligned}
$$

with

$$
\begin{aligned}
I_{1}^{a}(h) & =\int_{p} \frac{\ln \hat{p}^{2}}{\hat{p}^{2}+h}, \\
I_{1}^{b}(h) & =\int_{p} \frac{\left(\ln \hat{p}^{2}\right)^{2}}{\hat{p}^{2}+h} \\
I_{1}^{c}(h) & =\int_{p} \frac{1}{\hat{p}^{2}+h} \frac{\sum_{\mu} \sin ^{2} p_{\mu}}{\hat{p}^{2}}, \\
I_{1}^{d}(h) & =\int_{p} \frac{\ln \hat{p}^{2}}{\hat{p}^{2}+h} \frac{\sum_{\mu} \sin ^{2} p_{\mu}}{\hat{p}^{2}}, \\
I_{1}^{e}(h) & =\int_{p} \frac{1}{\hat{p}^{2}+h} \sum_{\mu, \nu} \frac{\sin ^{2} p_{\mu} \sin ^{2} p_{\nu}}{\left(\hat{p}^{2}\right)^{2}}, \\
I_{1}^{f}(h) & =\int_{p} \frac{1}{\hat{p}^{2}+h} \sum_{\mu} \tilde{F}_{\mu \mu}(p, 0), \\
I_{1}^{g}(h) & =\int_{p} \frac{\ln \hat{p}^{2}}{\hat{p}^{2}+h} \sum_{\mu} \tilde{F}_{\mu \mu}(p, 0), \\
I_{1}^{h}(h) & =\int_{p} \frac{1}{\hat{p}^{2}+h} \sum_{\mu, \nu} \tilde{F}_{\mu \nu}(p, 0) \frac{\sin p_{\mu} \sin p_{\nu}}{\hat{p}^{2}}, \\
I_{1}^{i}(h) & =\int_{p} \frac{1}{\hat{p}^{2}+h} \sum_{\mu, \nu} \tilde{F}_{\mu \nu}(p, 0)^{2} .
\end{aligned}
$$


The first five integrals diverge for small $h$. It is not difficult to first compute the divergent terms analytically and then evaluate the remaining constant terms numerically in the limit $h \rightarrow 0$. Our results are

$$
\begin{aligned}
& I_{1}^{a}(h)=-\frac{1}{8 \pi}(\ln h)^{2}+c_{1}+\mathcal{O}(h \ln h), \\
& I_{1}^{b}(h)=-\frac{1}{12 \pi}\left[(\ln h)^{3}+\pi^{2} \ln h\right]+c_{2}+\mathcal{O}(h \ln h), \\
& I_{1}^{c}(h)=K(h)-\frac{1}{\pi^{2}} c_{3}+\mathcal{O}(h \ln h), \\
& I_{1}^{d}(h)=-\frac{1}{8 \pi}(\ln h)^{2}+c_{4}+\mathcal{O}(h \ln h), \\
& I_{1}^{e}(h)=K(h)+c_{5}+\mathcal{O}(h \ln h),
\end{aligned}
$$

where the values for $c_{1}, \cdots, c_{5}$ are listed in Table 7 . The last four integrals in Eqs. (A.25)-(A.28) are, on the other hand, well-defined for $h \rightarrow 0$. Eqs. (A.25)(A.27) can be transformed to lattice sums in this limit

$$
\begin{aligned}
I_{1}^{f}(0) & =\sum_{x} G(x)\left[x^{2} H(x)^{2}-\frac{1}{4 \pi} \sum_{\mu}\left(\partial_{\mu}+\partial_{\mu}^{*}\right)^{2} G(x)+\frac{1}{\pi} H(x)\right], \\
I_{1}^{g}(0) & =\sum_{x} L(x)\left[x^{2} H(x)^{2}-\frac{1}{4 \pi} \sum_{\mu}\left(\partial_{\mu}+\partial_{\mu}^{*}\right)^{2} G(x)+\frac{1}{\pi} H(x)\right], \\
I_{1}^{h}(0) & =-\frac{1}{4} \sum_{x} G(x) \sum_{\mu, \nu} x_{\nu}\left[V_{\mu \nu}(x+\hat{\mu})-V_{\mu \nu}(x-\hat{\mu})\right]+\frac{C}{4},
\end{aligned}
$$

where the functions $L(x)$ and $V_{\mu \nu}(x)$ are defined by

$$
\begin{aligned}
L(x) & =\int_{p} \frac{\ln \hat{p}^{2}}{\hat{p}^{2}}\left(\mathrm{e}^{i p x}-1\right), \\
V_{\mu \nu}(x) & =x_{\mu} x_{\nu} H(x)^{2}-\frac{1}{4 \pi}\left(\partial_{\mu}+\partial_{\mu}^{*}\right)\left(\partial_{\nu}+\partial_{\nu}^{*}\right) G(x)+\frac{1}{2 \pi} \delta_{\mu \nu} H(x) .
\end{aligned}
$$

The numerical values for Eqs. (A.34)-( coordinate space technique are given in Table 0 . As for the last integral $I_{1}^{i}(0)$ of Eq. (A.28), we can compute it by a Fortran program calling NAGLIB integration subroutines. We get a number in Table 7 .

Let us now consider the integral $I_{2}(h)$ of Eq. (A.9). With help of the function

$$
H_{\mu \nu}(p, h)=F_{\mu \nu}(p, h)-F_{\mu \nu}(p, 0),
$$

it can be divided into two integrals

$$
I_{2}(h)=A(h)+2 B(h)
$$


with

$$
\begin{aligned}
A(h) & =\int_{p} \frac{1}{\hat{p}^{2}+h} \sum_{\mu, \nu} H_{\mu \nu}(p, h)^{2}, \\
B(h) & =\int_{p} \frac{1}{\hat{p}^{2}+h} \sum_{\mu, \nu} H_{\mu \nu}(p, h) F_{\mu \nu}(p, 0)
\end{aligned}
$$

which we would like to evaluate in the $h \rightarrow 0$ limit.

Noting that Eq. (A.41) has a finite limit for $h \rightarrow 0$, we perform the variable change $p \rightarrow \sqrt{h} q$ and take $h \rightarrow 0$ to transform to

$$
A(h) \stackrel{h \rightarrow 0}{=} \int_{-\infty}^{\infty} \frac{\mathrm{d}^{2} q}{(2 \pi)^{2}} \frac{1}{q^{2}+1} \sum_{\mu, \nu} \bar{H}_{\mu \nu}(q)^{2}
$$

with

$$
\begin{aligned}
\bar{H}_{\mu \nu}(q) & =\lim _{h \rightarrow 0} H_{\mu \nu}(q \sqrt{h}, h) \\
& =\int_{-\infty}^{\infty} \frac{\mathrm{d}^{2} q}{(2 \pi)^{2}} r_{\mu} s_{\nu}\left(\frac{1}{r^{2}+1} \frac{1}{s^{2}+1}-\frac{1}{r^{2} s^{2}}\right), \quad s=q-r
\end{aligned}
$$

which can exactly be evaluated by applying Feynman parametrization

$$
\bar{H}_{\mu \nu}(q)=\frac{1}{8 \pi} \delta_{\mu \nu}\left(-\ln q^{2}+\frac{2 \sqrt{q^{2}+4} \operatorname{arctanh} \sqrt{\frac{q^{2}}{q^{2}+4}}}{\sqrt{q^{2}}}\right)-\frac{q_{\mu} q_{\nu}}{\pi} \frac{\operatorname{arctanh} \sqrt{\frac{q^{2}}{q^{2}+4}}}{\left(q^{2}\right)^{3 / 2} \sqrt{q^{2}+4}} .
$$

By transforming to polar coordinates, the two-dimensional integral $A(0)$ of Eq. (A.43) can be reduced to an one-dimensional one being available to a numerical integration to high precision. Its result is reported in Table 7 .

The integral $B(h)$ from Eq. (A.42) is a little more tricky since it diverges for $h \rightarrow 0$. We apply Eq. (A.12) to rewrite

$$
B(h)=-\frac{1}{4}\left[B_{1}(h)+B_{2}(h)\right]
$$

where

$$
\begin{aligned}
& B_{1}(h)=\int_{p} \frac{1}{\hat{p}^{2}+h} \sum_{\mu, \nu} H_{\mu \nu}(p, h) \tilde{F}_{\mu \nu}(p, 0) \\
& B_{2}(h)=\int_{p} \frac{1}{\hat{p}^{2}+h} \sum_{\mu, \nu} H_{\mu \nu}(p, h)\left[-\frac{\delta_{\mu \nu}}{2 \pi} \ln \hat{p}^{2}-\frac{1}{\pi} \frac{\sin p_{\mu} \sin p_{\nu}}{\hat{p}^{2}}+\delta_{\mu \nu} C\right] \cdot(
\end{aligned}
$$

For the evaluation of $B_{1}(h)$, we perform the variable change $p \rightarrow \sqrt{h} q$ and let $h \rightarrow 0$. Then, we have

$$
B_{1}(h) \stackrel{h \rightarrow 0}{=} \int_{-\infty}^{\infty} \frac{\mathrm{d}^{2} q}{(2 \pi)^{2}} \frac{1}{q^{2}+1} \sum_{\mu, \nu} \bar{H}_{\mu \nu}(q) \tilde{F}_{\mu \nu}(0,0)
$$




\begin{tabular}{c|l||c|c}
\hline Constants & \multicolumn{1}{|c||}{ Values } & Constants & Values \\
\hline$c_{1}$ & $0.150666215804(1)$ & $I_{1}^{h}(0)$ & $-0.0165452709(1)$ \\
$c_{2}$ & $0.420051822207(1)$ & $I_{1}^{i}(0)$ & $0.0169938575(1)$ \\
$c_{3}$ & $1.682002936874882(1)$ & $A(0)$ & $0.001687475879587(1)$ \\
$c_{4}$ & $-0.0355227164897(1)$ & $B_{2}^{a}$ & $0.017838149659537(1)$ \\
$c_{5}$ & $-0.2327464349513382(1)$ & $B_{2}^{b}$ & $-0.006827009250335(1)$ \\
$I_{1}^{f}(0)$ & $-0.066086583814(1)$ & $B_{2}^{c}$ & $0.001497591836898(1)$ \\
$I_{1}^{g}(0)$ & $-0.07360068(1)$ & $B_{2}^{d}$ & $0.017838149659537(1)$ \\
\hline
\end{tabular}

Table 7: Numerical values for constant terms appearing in the determination of $W_{2}$.

which is identically zero. As for the integral $B_{2}(h)$, we do the same variable change $p \rightarrow \sqrt{h} q$. In the limit $h \rightarrow 0$, we get

$$
B_{2}(h) \stackrel{h \rightarrow 0}{=}-\frac{1}{2 \pi}(\ln h) B_{2}^{a}-\frac{1}{2 \pi} B_{2}^{b}-\frac{1}{\pi} B_{2}^{c}+C B_{2}^{d}
$$

with the two-dimensional, well-defined integrals

$$
\begin{aligned}
B_{2}^{a} & =\int_{-\infty}^{\infty} \frac{\mathrm{d}^{2} q}{(2 \pi)^{2}} \frac{1}{q^{2}+1} \sum_{\mu} \bar{H}_{\mu \mu}(q), \\
B_{2}^{b} & =\int_{-\infty}^{\infty} \frac{\mathrm{d}^{2} q}{(2 \pi)^{2}} \frac{\ln q^{2}}{q^{2}+1} \sum_{\mu} \bar{H}_{\mu \mu}(q), \\
B_{2}^{c} & =\int_{-\infty}^{\infty} \frac{\mathrm{d}^{2} q}{(2 \pi)^{2}} \frac{1}{q^{2}+1} \sum_{\mu, \nu} \bar{H}_{\mu \nu}(q) \frac{q_{\mu} q_{\nu}}{q^{2}}, \\
B_{2}^{d} & =\int_{-\infty}^{\infty} \frac{\mathrm{d}^{2} q}{(2 \pi)^{2}} \frac{1}{q^{2}+1} \sum_{\mu} \bar{H}_{\mu \mu}(q) .
\end{aligned}
$$

By transforming to polar coordinates, all of these integrals can again be reduced to one-dimensional ones whose numerical values are listed in Table 7 .

\section{A.2 Evaluation of $W_{1}$}

$W_{1}$ is defined as a finite part of an infrared divergent integral:

$$
W_{1}=\lim _{h \rightarrow 0}\left[\bar{W}_{1}(h)+\frac{1}{2} K(h)^{2}-\frac{3}{8 \pi} K(h)\right]
$$

with

$$
\bar{W}_{1}(h)=\int_{q r s} \frac{\sum_{\mu}\left(\hat{q}_{\mu}^{4}-2 \hat{q}_{\mu}^{2} \hat{r}_{\mu}^{2}\right)}{\left(\hat{q}^{2}+h\right)^{2}\left(\hat{r}^{2}+h\right)\left(\hat{s}^{2}+h\right)} \bar{\delta}(q+r+s) .
$$


Our aim is again to evaluate the integral $\bar{W}_{1}(h)$ in the limit $h \rightarrow 0$ to confirm the logarithmic terms in Eq. (A.55) and to determine the constant $W_{1}$ at the end.

Eq. (A.56) can be transformed to a symmetric form

$$
\bar{W}_{1}(h)=\int_{q r s} \frac{\sum_{\mu} \hat{q}_{\mu}^{2}\left[(r+s)_{\mu}^{2}-\hat{r}_{\mu}^{2}-\hat{s}_{\mu}^{2}\right]}{\left(\hat{q}^{2}+h\right)^{2}\left(\hat{r}^{2}+h\right)\left(\hat{s}^{2}+h\right)} \bar{\delta}(q+r+s)
$$

which can, by expanding the numerator, be divided into two integrals

$$
\bar{W}_{1}(h)=2 T_{1}(h)-\frac{1}{2} T_{2}(h)
$$

with

$$
\begin{aligned}
& T_{1}(h)=\int_{q r s} \frac{\sum_{\mu} \hat{q}_{\mu}^{2} \sin r_{\mu} \sin s_{\mu}}{\left(\hat{q}^{2}+h\right)^{2}\left(\hat{r}^{2}+h\right)\left(\hat{s}^{2}+h\right)} \bar{\delta}(q+r+s), \\
& T_{2}(h)=\int_{q r s} \frac{\sum_{\mu} \hat{q}_{\mu}^{2} \hat{r}_{\mu}^{2} \hat{s}_{\mu}^{2}}{\left(\hat{q}^{2}+h\right)^{2}\left(\hat{r}^{2}+h\right)\left(\hat{s}^{2}+h\right)} \bar{\delta}(q+r+s) .
\end{aligned}
$$

Noting that Eq. (A.59) can, with help of Eq. (A.6), be represented by

$$
T_{1}(h)=\sum_{\mu} \int_{q} \frac{\hat{q}_{\mu}^{2}}{\left(\hat{q}^{2}+h\right)^{2}} F_{\mu \mu}(q, h),
$$

we can evaluate it analogously to Eq. (A.5). We break up Eq. (A.61) into two parts

$$
T_{1}(h)=T_{1}^{a}(h)+T_{1}^{b}(h),
$$

where

$$
\begin{aligned}
T_{1}^{a}(h) & =\sum_{\mu} \int_{q} \frac{\hat{q}_{\mu}^{2}}{\left(\hat{q}^{2}+h\right)^{2}} F_{\mu \mu}(q, 0), \\
T_{1}^{b}(h) & =\sum_{\mu} \int_{q} \frac{\hat{q}_{\mu}^{2}}{\left(\hat{q}^{2}+h\right)^{2}}\left[F_{\mu \mu}(q, h)-F_{\mu \mu}(q, 0)\right] .
\end{aligned}
$$

By analogy to $I_{1}(h)$ of Eq. (A.8), Eq. (A.63) can be expanded to

$$
T_{1}^{a}(h)=-\frac{1}{4} \sum_{\mu} \int_{q} \frac{\hat{q}_{\mu}^{2}}{\left(\hat{q}^{2}+h\right)^{2}}\left[\tilde{F}_{\mu \mu}(q, 0)-\frac{1}{\pi} \frac{\sin ^{2} q_{\mu}}{\hat{q}^{2}}-\frac{1}{2 \pi} \delta_{\mu \nu} \ln \hat{q}^{2}+C\right]
$$

which produces the following four integrals being able to be evaluated separately:

$$
T_{1}^{a}(h)=-\frac{1}{4}\left[C J_{1}(h)-\frac{1}{2 \pi} J_{2}(h)-\frac{1}{\pi} J_{3}(h)+J_{4}(h)\right]
$$


with

$$
\begin{aligned}
& J_{1}(h)=\int_{q} \frac{\hat{q}^{2}}{\left(\hat{q}^{2}+h\right)^{2}}, \\
& J_{2}(h)=\int_{q} \frac{\hat{q}^{2}}{\left(\hat{q}^{2}+h\right)^{2}} \ln \hat{q}^{2}, \\
& J_{3}(h)=\sum_{\mu} \int_{q} \frac{\hat{q}_{\mu}^{2}}{\left(\hat{q}^{2}+h\right)^{2}} \frac{\sin ^{2} q_{\mu}}{\hat{q}^{2}}, \\
& J_{4}(h)=\sum_{\mu} \int_{q} \frac{\hat{q}_{\mu}^{2}}{\left(\hat{q}^{2}+h\right)^{2}} \tilde{F}_{\mu \mu}(q, 0) .
\end{aligned}
$$

The first three integrals diverge for $h \rightarrow 0$. After having isolated the divergent parts analytically, the remaining constant terms can be determined numerically:

$$
\begin{aligned}
& J_{1}(h)=K(h)-\frac{1}{4 \pi}+\mathcal{O}(h \ln h), \\
& J_{2}(h)=-\frac{1}{8 \pi}\left[(\ln h)^{2}+2 \ln h\right]+k_{1}+\mathcal{O}(h \ln h), \\
& J_{3}(h)=-\frac{3}{16 \pi} \ln h+k_{2}+\mathcal{O}(h \ln h),
\end{aligned}
$$

where the values for $k_{1}$ and $k_{2}$ are given in Table 8. The last integral $J_{4}(h)$ is, on the other hand, infrared finite and can, in the $h \rightarrow 0$ limit, be transformed to a lattice sum

$$
J_{4}(0)=-\sum_{x} \sum_{\mu}\left[\left(\partial_{\mu}-\partial_{\mu}^{*}\right) G_{2}(x)\right]\left[x_{\mu}^{2} H(x)^{2}-\frac{1}{4 \pi}\left(\partial_{\mu}+\partial_{\mu}^{*}\right)^{2} G(x)+\frac{1}{2 \pi} H(x)\right],
$$

where the function $G_{2}(x)$ including its properties is discussed in detail in Ref. 16. The number of $J_{4}(0)$ computed by the position space technique is shown in Table 8 .

Concerning the integral $T_{1}^{b}(h)$ of Eq. (A.64), it can be expressed by

$$
T_{1}^{b}(h)=\sum_{\mu} \int_{q} \frac{\hat{q}_{\mu}^{2}}{\left(\hat{q}^{2}+h\right)^{2}} H_{\mu \mu}(q, h)
$$

with $H_{\mu \mu}(q, h)$ from Eq. (A.39). By changing the variable $q=\sqrt{h} p$ and taking the limit $h \rightarrow 0$, Eq. (A.75) becomes

$$
T_{1}^{b}(0)=\sum_{\mu} \int_{-\infty}^{\infty} \frac{\mathrm{d}^{2} p}{(2 \pi)^{2}} \frac{p_{\mu}^{2}}{\left(p^{2}+1\right)^{2}} \bar{H}_{\mu \mu}(p) .
$$

By transforming to polar coordinates, this two-dimensional integral can be reduced to one-dimensional one whose numerical value is reported in Table 8 . 


\begin{tabular}{c|c||c|c}
\hline Constants & Values & Constants & Values \\
\hline$k_{1}$ & $0.150666215804(1)$ & $J_{4}(0)$ & $-0.057000592735(1)$ \\
$k_{2}$ & $0.00658179485930(1)$ & $T_{1}^{b}(0)$ & $0.001497591836898(1)$ \\
$k_{3}$ & $0.68169011381621(1)$ & $T_{2}^{a}(0)$ & $-0.02533029591(1)$ \\
\hline
\end{tabular}

Table 8: Numerical values for constant terms appearing in the determination of $W_{1}$.

The last integral $T_{2}(h)$ of Eq. (A.60) is to be evaluated by dividing into two parts

$$
T_{2}(h)=T_{2}^{a}(h)+T_{2}^{b}(h),
$$

where

$$
\begin{aligned}
T_{2}^{a}(h) & =\int_{q r s} \frac{\sum_{\mu} \hat{q}_{\mu}^{2} \hat{r}_{\mu}^{2} \hat{s}_{\mu}^{2}}{\left(\hat{q}^{2}+h\right)^{2}\left(\hat{r}^{2}+h\right)\left(\hat{s}^{2}+h\right)}[\bar{\delta}(q+r+s)-\bar{\delta}(r+s)], \\
T_{2}^{b}(h) & =\sum_{\mu} \int_{q} \frac{\hat{q}_{\mu}^{2}}{\left(\hat{q}^{2}+h\right)^{2}}\left[\int_{r s} \frac{\hat{r}_{\mu}^{2} \hat{s}_{\mu}^{2}}{\left(\hat{r}^{2}+h\right)\left(\hat{s}^{2}+h\right)} \bar{\delta}(r+s)\right] .
\end{aligned}
$$

In the limit $h \rightarrow 0$, Eq. (A.78) is finite and can be transformed to a lattice sum

$$
T_{2}^{a}(0)=-\sum_{x} \sum_{\mu}\left[\left(\partial_{\mu}-\partial_{\mu}^{*}\right) G_{2}(x)\right]\left[\left(\partial_{\mu}-\partial_{\mu}^{*}\right) G(x)\right]^{2},
$$

while Eq. (A.79) reduces to

$$
T_{2}^{b}(h)=\frac{1}{2} k_{3}\left[K(h)-\frac{1}{4 \pi}\right]+\mathcal{O}(h \ln h)
$$

with

$$
k_{3}=\int_{r} \frac{1}{\left(\hat{r}^{2}\right)^{2}} \sum_{\mu} \hat{r}_{\mu}^{4}
$$

The numerical values for $T_{2}^{a}(0)$ and $k_{3}$ are listed in Table 8 .

\section{A.3 Evaluation of the constant lattice integrals}

The constant lattice integrals appearing in Table 1 of section 2 are defined as follows:

$$
\begin{aligned}
G_{1} & =-\frac{1}{4} \int_{q r s} \frac{\hat{q}^{2}-\hat{r}^{2}-\hat{s}^{2}}{\hat{r}^{2} \hat{s}^{2}} \frac{\sum_{\mu} \hat{q}_{\mu}^{4}}{\left(\hat{q}^{2}\right)^{2}} \bar{\delta}(q+r+s), \\
J & =\int_{q r s t} \frac{\left(\sum_{\mu} \hat{q}_{\mu} \hat{r}_{\mu} \hat{s}_{\mu} \hat{t}_{\mu}\right)^{2}}{\hat{q}^{2} \hat{r}^{2} \hat{s}^{2} \hat{t}^{2}} \bar{\delta}(q+r+s+t),
\end{aligned}
$$




$$
\begin{aligned}
& K=\int_{q r s t} \frac{\left[(\widehat{q+r})^{2}-\hat{q}^{2}-\hat{r}^{2}\right]\left[(\widehat{s+t})^{2}-\hat{s}^{2}-\hat{t}^{2}\right]}{\hat{q}^{2} \hat{r}^{2} \hat{s}^{2} \hat{t}^{2}} \bar{\delta}(q+r+s+t), \\
& L_{1}=\int_{q r s t} \frac{\sum_{\mu, \nu} \hat{q}_{\mu} \hat{q}_{\nu} \hat{s}_{\mu} \hat{s}_{\nu} \hat{t}_{\mu} \hat{t}_{\nu}\left[\hat{r}_{\mu} \hat{r}_{\nu}(\widehat{s+t})^{2}-(\widehat{s+t})_{\mu}(\widehat{s+t})_{\nu} \hat{r}^{2}\right]}{\left(\hat{q}^{2}\right)^{2} \hat{r}^{2} \hat{s}^{2} \hat{t}^{2}(\widehat{s+t})^{2}} \bar{\delta}(q+r+s+t), \\
& V_{1}=\int_{q r s t} \frac{\sum_{\mu} \hat{q}_{\mu} \hat{r}_{\mu} \hat{s}_{\mu} \hat{t}_{\mu}}{\hat{q}^{2} \hat{r}^{2} \hat{s}^{2} \hat{t}^{2}} \bar{\delta}(q+r+s+t) \\
& V_{2}=\int_{q r s t} \frac{\left[(\widehat{s+t})^{2}-\hat{s}^{2}-\hat{t}^{2}\right]}{\hat{s}^{2} \hat{t}^{2}} \sum_{\mu} \frac{\left[\frac{1}{2}(q \widehat{+r})_{\mu}^{2} \hat{q}^{2}+(q \widehat{+r})^{2} \hat{q}_{\mu}^{2}\right]}{\left(\hat{q}^{2}\right)^{2} \hat{r}^{2}} \\
& \times \frac{\left[\left(\hat{q}_{\mu}^{2}+\hat{r}_{\mu}^{2}\right)(q \widehat{+r})^{2}-\left(\hat{q}^{2}+\hat{r}^{2}\right)(q \widehat{+r})_{\mu}^{2}\right]}{\left[(q \widehat{+r})^{2}\right]^{2}} \bar{\delta}(q+r+s+t), \\
& V_{3}=\int_{q r s t} \frac{\left[(\widehat{s+t})^{2}-\hat{s}^{2}-\hat{t}^{2}\right]\left[(\widehat{q+r})^{2}-\hat{q}^{2}-\hat{r}^{2}\right]}{\hat{q}^{2} \hat{r}^{2} \hat{s}^{2} \hat{t}^{2}\left[(q \widehat{+r})^{2}\right]^{2}} \sum_{\mu}(q \widehat{+r})_{\mu}^{4} \bar{\delta}(q+r+s+t), \\
& V_{4}=\int_{q r s t} \frac{\sum_{\mu, \nu} \hat{r}_{\mu}^{2} \hat{t}_{\mu}^{2} \sin q_{\nu} \sin s_{\nu}}{\hat{q}^{2} \hat{r}^{2} \hat{s}^{2} \hat{t}^{2}(q+r)^{2}} \bar{\delta}(q+r+s+t) \\
& V_{5}=\int_{q r s t} \frac{\sum_{\mu, \nu} \hat{q}_{\mu} \hat{r}_{\mu} \hat{s}_{\mu} \hat{t}_{\mu} \sin ^{2}(q+r)_{\nu}}{\hat{q}^{2} \hat{r}^{2} \hat{s}^{2} \hat{t}^{2}(q+r)^{2}} \bar{\delta}(q+r+s+t) \\
& V_{6}=\int_{q r s t} \frac{\left[(\widehat{s+t})^{2}-\hat{s}^{2}-\hat{t}^{2}\right] \sum_{\mu}(q \widehat{+r})_{\mu}^{2} \hat{q}_{\mu} \hat{r}_{\mu} \cos \frac{1}{2}(q+r)_{\mu}}{\hat{q}^{2} \hat{r}^{2} \hat{s}^{2} \hat{t}^{2}(q \widehat{+r})^{2}} \bar{\delta}(q+r+s+t) \text {. }
\end{aligned}
$$

We can transform all of these integrals except $V_{4}$ and $V_{5}$ to position space and then apply the coordinate space method [16]:

$$
\begin{aligned}
G_{1} & =\frac{1}{8} \sum_{x}\left\{\sum_{\mu} \partial_{\mu}^{*} \partial_{\mu} \partial_{\mu}^{*} \partial_{\mu} G_{2}(x)\right\}\left\{\sum_{\mu}\left[\left(\left(\partial_{\mu}-\partial_{\mu}^{*}\right) G(x)\right)^{2}+\left(\left(\partial_{\mu}+\partial_{\mu}^{*}\right) G(x)\right)^{2}\right]\right\} \\
J & =\sum_{x} \sum_{\mu, \nu}\left[\partial_{\mu} \partial_{\nu} G(x)\right]^{4} \\
K & =\frac{1}{4} \sum_{x}\left\{\sum_{\mu}\left[\left(\left(\partial_{\mu}-\partial_{\mu}^{*}\right) G(x)\right)^{2}+\left(\left(\partial_{\mu}+\partial_{\mu}^{*}\right) G(x)\right)^{2}\right]\right\}^{2}, \\
L_{1} & =\sum_{x} \sum_{\mu, \nu}\left[\partial_{\mu} \partial_{\nu} G_{2}(x)\right]\left[\partial_{\mu} \partial_{\nu} G(x)\right]^{3} \\
V_{1} & =\sum_{x} \sum_{\mu}\left[\partial_{\mu} G(x)\right]^{4} \\
V_{2} & =-\frac{1}{2} \sum_{x}\left\{\sum_{\mu}\left[\left(\partial_{\mu}-\partial_{\mu}^{*}\right) G(x)\right]^{2}+\sum_{\mu}\left[\left(\partial_{\mu}+\partial_{\mu}^{*}\right) G(x)\right]^{2}\right\}
\end{aligned}
$$




$$
\begin{aligned}
& \times\left\{G(x) \sum_{\mu}\left(\partial_{\mu}-\partial_{\mu}^{*}\right)^{2} G_{2}(x)+\sum_{\mu}\left[\left(\partial_{\mu}-\partial_{\mu}^{*}\right) G(x)\right]\left[\left(\partial_{\mu}-\partial_{\mu}^{*}\right) G_{2}(x)\right]\right\}, \\
V_{3}= & \sum_{x} G(x)^{2} \sum_{\mu}\left(\partial_{\mu}-\partial_{\mu}^{*}\right)^{2} G(x)^{2}, \\
V_{6}= & \frac{1}{8} \sum_{x} G(x)^{2} \sum_{\mu}\left\{2\left[\left(\partial_{\mu}-\partial_{\mu}^{*}\right)\left(\partial_{\mu}+\partial_{\mu}^{*}\right) G(x)\right][G(x+2 \hat{\mu})-G(x-2 \hat{\mu})]\right. \\
& \left.+\left[\left(\partial_{\mu}+\partial_{\mu}^{*}\right)^{2} G(x)\right]\left[\left(\partial_{\mu}-\partial_{\mu}^{*}\right)^{2} G(x)+\left(\partial_{\mu}+\partial_{\mu}^{*}\right)^{2} G(x)\right]\right\} .
\end{aligned}
$$

Concerning the integral $V_{4}$, after transforming to a symmetric form

$$
V_{4}=-\int_{t} \frac{1}{\hat{t}^{2}} \sum_{\mu, \nu} A_{\mu \nu}(t)^{2}
$$

with

$$
A_{\mu \nu}(t)=\int_{q} \frac{(t \widehat{+q})_{\mu}^{2} \sin q_{\nu}}{(t \widehat{+q})^{2} \hat{q}^{2}},
$$

we can compute it by a Fortran program calling NAGLIB integration subroutines. The integral $V_{5}$ can, on the other hand, be divided into

$$
V_{5}=V_{5}^{a}-\frac{1}{4} V_{5}^{b}
$$

where

$$
\begin{aligned}
V_{5}^{a} & =\int_{q r s t} \frac{\sum_{\mu} \hat{q}_{\mu} \hat{r}_{\mu} \hat{s}_{\mu} \hat{t}_{\mu}}{\hat{q}^{2} \hat{r}^{2} \hat{s}^{2} \hat{t}^{2}} \bar{\delta}(q+r+s+t), \\
V_{5}^{b} & =\int_{q r s t} \frac{\sum_{\mu} \hat{q}_{\mu} \hat{r}_{\mu} \hat{s}_{\mu} \hat{t}_{\mu} \sum_{\nu}(q \widehat{+r})_{\nu}^{4}}{\hat{q}^{2} \hat{r}^{2} \hat{s}^{2} \hat{t}^{2}(q+r)^{2}} \bar{\delta}(q+r+s+t) .
\end{aligned}
$$

$V_{5}^{a}$ is then able to be represented in coordinate space

$$
V_{5}^{a}=\sum_{x} \sum_{\mu}\left[\partial_{\mu} G(x)\right]^{4}
$$

while $V_{5}^{b}$ can be symmetrized as

$$
V_{5}^{b}=\int_{t} \frac{\sum_{\mu} \hat{t}_{\mu}^{4}}{\hat{t}^{2}} \sum_{\nu} B_{\nu}(t)^{2}
$$

with

$$
B_{\nu}(t)=\int_{q} \frac{(t \widehat{+q})_{\nu} \hat{q}_{\nu}}{(t \widehat{+q})^{2} \hat{q}^{2}}
$$

which is again to be evaluated by using NAGLIB integration subroutines. 


\section{References}

[1] M. Lüscher, Nucl. Phys. B 135 (1978) 1.

[2] M. Lüscher, Addendum to Ref. [1] (1986), unpublished notes.

[3] D. Buchholz and J.T. Lopuszański, Lett. Math. Phys. 3 (1979) 175.

[4] D. Buchholz and J.T. Lopuszański, Nucl. Phys. B 263 (1986) 155.

[5] A.B. Zamolodchikov and A.B. Zamolodchikov, Ann. Phys. (NY) 120 (1979) 253.

[6] A.B. Zamolodchikov and A.B. Zamolodchikov, Nucl. Phys. B 133 (1978) 525.

[7] P. Hasenfratz, M. Maggiore and F. Niedermayer, Phys. Lett. B 245 (1990) 522.

[8] P. Hasenfratz and F. Niedermayer, Phys. Lett. B 245 (1990) 529.

[9] N. D. Mermin and H. Wagner, Phys. Rev. Lett. 17 (1966) 1133.

[10] A. Patrascioiu and E. Seiler, J. Stat. Phys. 69 (1992) 573.

[11] A. Patrascioiu and E. Seiler, Nucl. Phys. B (Proc. Suppl.) 30 (1993) 184.

[12] A. Patrascioiu and E. Seiler, Phys. Lett. B 430 (1998) 314.

[13] J. M. Kosterlitz and D. J. Thouless, J. Phys. C 6 (1973) 1181.

[14] J. M. Kosterlitz, J. Phys. C 7 (1974) 1046.

[15] S. Caracciolo and A. Pelissetto, Nucl. Phys. B 455 [FS] (1995) 619.

[16] D.-S. Shin, Nucl. Phys. B 525 (1998) 457.

[17] D.-S. Shin, Nucl. Phys. B 496 (1997) 408.

[18] B. Allés, G. Cella, M. Dilaver and Y. Gündüç, hep-lat/9808003.

[19] B. Allés, A. Buonanno and G. Cella, Nucl. Phys. B 500 (1997) 513.

[20] P. Butera and M. Comi, Phys. Rev. B 54 (1996) 15828.

[21] T. Mendes, A. Pelissetto and A. D. Sokal, Nucl. Phys. B 477 (1996) 203.

[22] D.-S. Shin, work in progress.

[23] S. Elitzur, Phys. Rev. D 12 (1975) 3978.

[24] F. David, Commun. Math. Phys. 81 (1981) 149. 
[25] E. Brézin and S. Hikami, J. Phys. A 11 (1976) 1141.

[26] S. Hikami, Phys. Lett. B 98 (1981) 208; Nucl. Phys. B 215 [FS7] (1983) 555.

[27] W. Bernreuther and F. J. Wegner, Phys. Rev. Lett. 57 (1986) 1383.

[28] F. J. Wegner, Nucl. Phys. B 316 (1989) 663.

[29] M. Falcioni and A. Treves, Nucl. Phys. B 265 [FS15] (1986) 671.

[30] P. Weisz, unpublished notes, cited in Ref. [34].

[31] S. Caracciolo and A. Pelissetto, Nucl. Phys. B 420 (1994) 141.

[32] P. Biscari, M. Campostrini and P. Rossi, Phys. Lett. B 242 (1990) 225.

[33] M. Campostrini and P. Rossi, Phys. Lett. B 242 (1990) 81.

[34] M. Lüscher, P. Weisz and U. Wolff, Nucl. Phys. B 359 (1991) 221.

[35] B. Allés, S. Caracciolo, A. Pelissetto and M. Pepe, Nucl. Phys. B 562 (1999) 581.

[36] D.-S. Shin and O. Veretin, in preparation. 